\title{
Mesenchymal stem cell transplantation in tight-skin mice identifies miR-151-5p as a therapeutic target for systemic sclerosis
}

Chider Chen ${ }^{1}$, Dandan Wang ${ }^{2}$, Alireza Moshaverinia ${ }^{3}$, Dawei Liu ${ }^{1}$, Xiaoxing Kou ${ }^{1}$, Wenjing Yu ${ }^{1}$, Ruili Yang ${ }^{1}$, Lingyun Sun ${ }^{2}$, Songtao Shi ${ }^{1}$

${ }^{I}$ Department of Anatomy and Cell Biology, University of Pennsylvania, School of Dental Medicine, Philadelphia, PA 19104, USA; ${ }^{2}$ Department of Rheumatology and Immunology, The Affiliated Drum Tower Hospital of Nanjing University Medical School, 321 Zhongshan Road, Nanjing, Jiangsu 210008, China; ${ }^{3}$ Division of Advanced Prosthodontics, Weintraub Center for Reconstructive Biotechnology, School of Dentistry, University of California, Los Angeles, CA 90095, USA

Systemic sclerosis (SSc), an autoimmune disease, may cause significant osteopenia due to activation of the IL4Ro/ mTOR pathway. Mesenchymal stem cell transplantation (MSCT) can ameliorate immune disorders in SSc via inducing immune tolerance. However, it is unknown whether MSCT rescues osteopenia phenotype in SSc. Here we show that MSCT can effectively ameliorate osteopenia in SSc mice by rescuing impaired lineage differentiation of the recipient bone marrow MSCs. Mechanistically, we show that donor MSCs transfer miR-151-5p to the recipient bone marrow MSCs in SSc mice to inhibit IL4R $\alpha$ expression, thus downregulating mTOR pathway activation to enhance osteogenic differentiation and reduce adipogenic differentiation. Moreover, systemic delivery of miR-151-5p is capable of rescuing osteopenia, impaired bone marrow MSCs, tight skin, and immune disorders in SSc mice, suggesting that miR-151-5p may be a specific target for SSc treatment. Our finding identifies a previously unrecognized role of MSCT in transferring miRNAs to recipient stem cells to ameliorate osteopenia via rescuing a non-coding RNA pathway.

Keywords: mesenchymal stem cell; systemic sclerosis; osteopenia; microRNA-151-5p; exosome

Cell Research (2017) 27:559-577. doi:10.1038/cr.2017.11; published online publication 20 January 2017

\section{Introduction}

Systemic sclerosis/scleroderma ( $\mathrm{SSc}$ ) is a connective tissue disorder characterized by progressive fibrosis of the skin, internal organ dysfunction, vasculopathy, and autoimmune rheumatic conditions [1]. Osteopenia of the vertebrae and other bones are commonly observed in SSc patients [2-6]. It has been suggested that bone loss in SSc patients may be due to immobilization, and medical treatments such as use of corticosteroids [7]. However, the detailed pathophysiological mechanism of bone de-

Correspondence: Songtao $\mathrm{Shi}^{\mathrm{a}}$, Lingyun Sun ${ }^{\mathrm{b}}$

${ }^{a}$ Tel: 215-898-3326; Fax: 215-573-5032

E-mail: songtaos@dental.upenn.edu

${ }^{\mathrm{b}}$ E-mail: lingyunsun2012@163.com

Received 11 May 2016; revised 3 October 2016; accepted 6 December

2016; published online publication 20 January 2017 fects in SSc patients is largely unknown.

Fibrillin-1 (Fbn1)-mutant tight-skin mice (B6.Cg-Fbn1Tsk/J; $T s k /^{+}$), an established SSc model, show significantly reduced femoral bone mineral density (BMD) and abnormal trabecular bone microarchitecture [8]. Recent studies indicate that type 2 helper T-cell $\left(\mathrm{T}_{\mathrm{H}} 2\right)$ infiltration and interleukin-4 (IL4), a key $\mathrm{T}_{\mathrm{H}} 2$ cytokine, play a critical role in the regulation of fibrotic tissue deposition and bone marrow mesenchymal stem cells (BMMSCs) [9]. Downregulation of the IL4/IL4R $\alpha$ signaling pathway in $T s k{ }^{+}$mice can rescue fibrotic and osteoporotic phenotypes, suggesting that IL4 signaling contributes to the pathological process in SSc $[10,11]$.

Systemic MSC transplantation (MSCT) has been used to treat a variety of human diseases such as graft versus host disease (GvHD), systemic lupus erythematosus (SLE), diabetes, rheumatoid arthritis, myocardial infarction, liver fibrosis, inflammatory bowel disease, 
autoimmune encephalomyelitis, periodontitis, sepsis and scleroderma [12-23]. At the molecular level, MSCT may employ a paracrine cytokine secretion mechanism to regulate recipient immune response, thereby achieving therapeutic effects [24-27]. In addition, MSCT is able to induce immune tolerance and ameliorate autoimmune phenotypes in SSc through activation of the Fas/Fas ligand pathway, leading to apoptosis of activated $\mathrm{T}$ cells $[28,29]$. However, it is unknown whether MSCT ameliorates the osteoporotic phenotype and rescues impaired BMMSCs in SSc.

MicroRNAs (miRNAs) are small non-coding RNAs that function as post-transcriptional regulators of gene expression [30]. It has been shown that coding and non-coding RNAs can be transferred between cells by small vesicles called exosomes [31-33]. Releasing large amounts of exosomes is one mean by which MSCs communicate with surrounding cells, implying that exosome-mediated cellular content transfer may serve as a novel mechanism offering therapeutic effects in MSCT $[34,35]$. In this study, we show that MSCT transfers miR-151-5p to $T s k /^{+}$recipients, rescuing impaired lineage differentiation of BMMSCs and osteopenia via regulating the IL4R $\alpha / \mathrm{mTOR}$ pathway.

\section{Results}

MSCT rescues osteopenia in $T s k{ }^{+}$mice by regulating osteo-/ adipo-genic lineage differentiation of recipient BMMSCs

$T_{s} k /^{+} \mathrm{SSc}$ mice exhibit significantly reduced bone volume and increase of bone marrow adipocytes [8, 11]. Since MSCT is able to ameliorate the osteoporotic phenotypes observed in SLE and ovariectomized (OVX) mice [13, 36, 37], we hypothesized that this therapeutic effect could extend to SSc. We therefore used allogeneic MSCT to treat $T_{s} k /^{+}$mice at 8 weeks of age and examined the therapeutic effects at 16 weeks of age (Figure 1A). MicroCT and histological analysis confirmed that BMD and distal femoral trabecular bone volume of $T s k /^{+}$ mice were markedly decreased compared with the control wild-type (WT) littermates (Figure 1B-1D). BMMSC transplantation (MSCT) significantly rescued BMD and trabecular bone volume in $T_{s k} /^{+}$mice (Figure 1B-1D). A histomorphometric analysis indicated that osteoblast and osteoclast numbers in the femurs of $T s k{ }^{+}$mice using alkaline phosphatase (ALP) immunohistochemical and tartrate-resistant acid phosphatase (TRAP) staining, respectively, were significantly reduced when compared to the control littermates (Figure 1E and $1 \mathrm{~F}$ ). MSCT significantly restored osteoblast and osteoclast numbers in the femurs of $T s k I^{+}$mice (Figure $1 \mathrm{E}$ and $1 \mathrm{~F}$ ). In addition, serum levels of soluble receptor activator of nuclear factor $\kappa \mathrm{B}$ ligand (sRANKL) and type I collagen cross-linked telopeptide (CTX) were significantly reduced in $T s k l^{+}$ mice (Figure $1 \mathrm{G}$ and $1 \mathrm{H}$ ). MSCT markedly improved the serum levels of CTX and sRANKL (Figure $1 \mathrm{G}$ and $1 \mathrm{H}$ ), suggesting that bone volume loss is mainly caused by insufficient osteoblast function in $T s k /^{+}$mice. To further confirm this observation, we performed a calcein-labeling assay to show that bone turnover rate was decreased in $T s k /^{+}$mice, whereas MSCT significantly improved bone formation rate (Figure 1I).

To examine whether MSCT rescued BMMSC lineage differentiation in $T s k /^{+}$mice, we isolated BMMSCs from MSCT-treated and untreated $T_{s} k /^{+}$mice. Our analysis revealed that the number of colony forming unit-fibroblasts (CFU-F) was significantly reduced in $T s k /^{+}$mice compared to the control group, while MSCT significantly elevated the numbers of CFU-F (Figure 2A). When cultured under osteogenic induction conditions, $T s k /^{+}$ BMMSCs showed impaired osteogenic differentiation, as indicated by reduced mineralized nodule formation (assessed by alizarin red staining) and reduced expression of the osteogenic genes runt-related transcription factor 2 (Runx2), $A L P$, and osteocalcin (OCN) (Figure $2 \mathrm{~B}$ and $2 \mathrm{C}$ ). MSCT was able to rescue impaired osteogenic differentiation of BMMSCs from $T s k /^{+}$mice, as observed by increased mineralized nodule formation and expression of Runx2, ALP, and $O C N$ (Figure 2B and 2C). We next showed that BMMSCs derived from $T s k l^{+}$mice generated less new bone than BMMSCs from the control group at 8 weeks post implantation using an established in vivo BMMSC implantation assay, in which $4 \times 10^{6}$ BMMSCs with hydroxyapatite tricalcium phosphate (HA/TCP) particles as a carrier were subcutaneously implanted into immunocompromised mice (Figure 2D). The impaired $T s k /^{+}$BMMSCs were rescued by MSCT as indicated by increased in vivo bone formation (Figure 2D). In addition to impaired osteogenesis, $T s k /^{+}$mice showed a markedly increased number of adipocytes in the bone marrow (Figure 2E). MSCT largely diminished numbers of adipocytes in the bone marrow of $T_{s} \mathrm{k} /{ }^{+}$mice (Figure 2E). When cultured under adipogenic induction conditions, $T s k /^{+}$BMMSCs showed increased numbers of Oil red O-positive cells and upregulation of adipogenic genes peroxisome proliferator-activated receptor gamma $2(P P A R \gamma)$ and lipoprotein lipase $(L P L)$; MSCT significantly reduced the number of Oil red O-positive cells and expression levels of PPAR $\gamma$ and $L P L$ in $T s k /^{+}$ BMMSCs (Figure $2 \mathrm{~F}$ and $2 \mathrm{G}$ ). Based on these results, we conclude that the osteopenia in $T s k l^{+}$mice is associated with altered lineage differentiation of BMMSCs, resulting in a decreased rate of bone formation. MSCT successfully ameliorates osteopenia in SSc mice via res- 

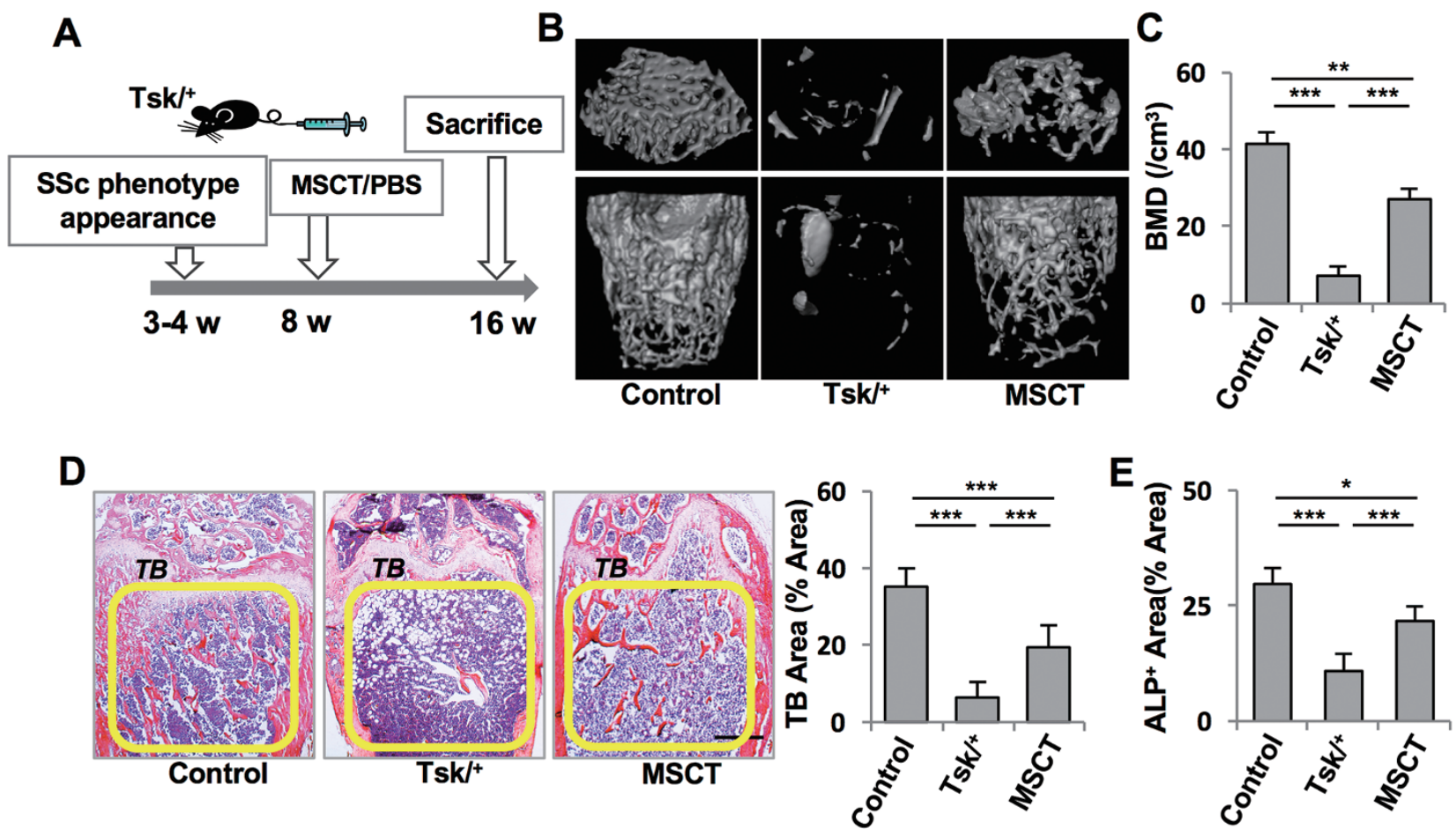

F

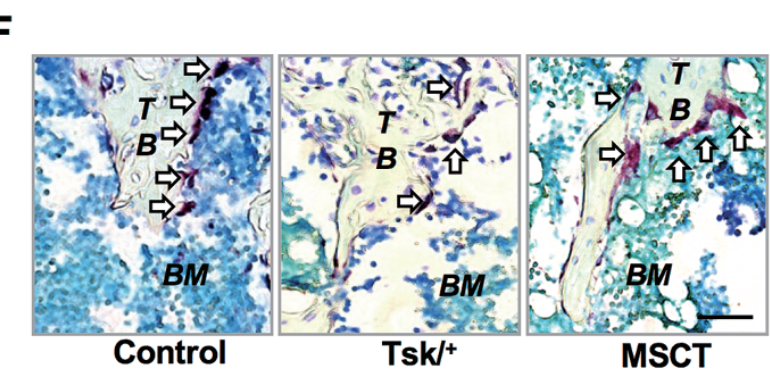

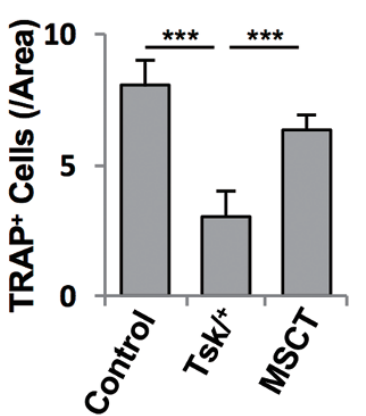
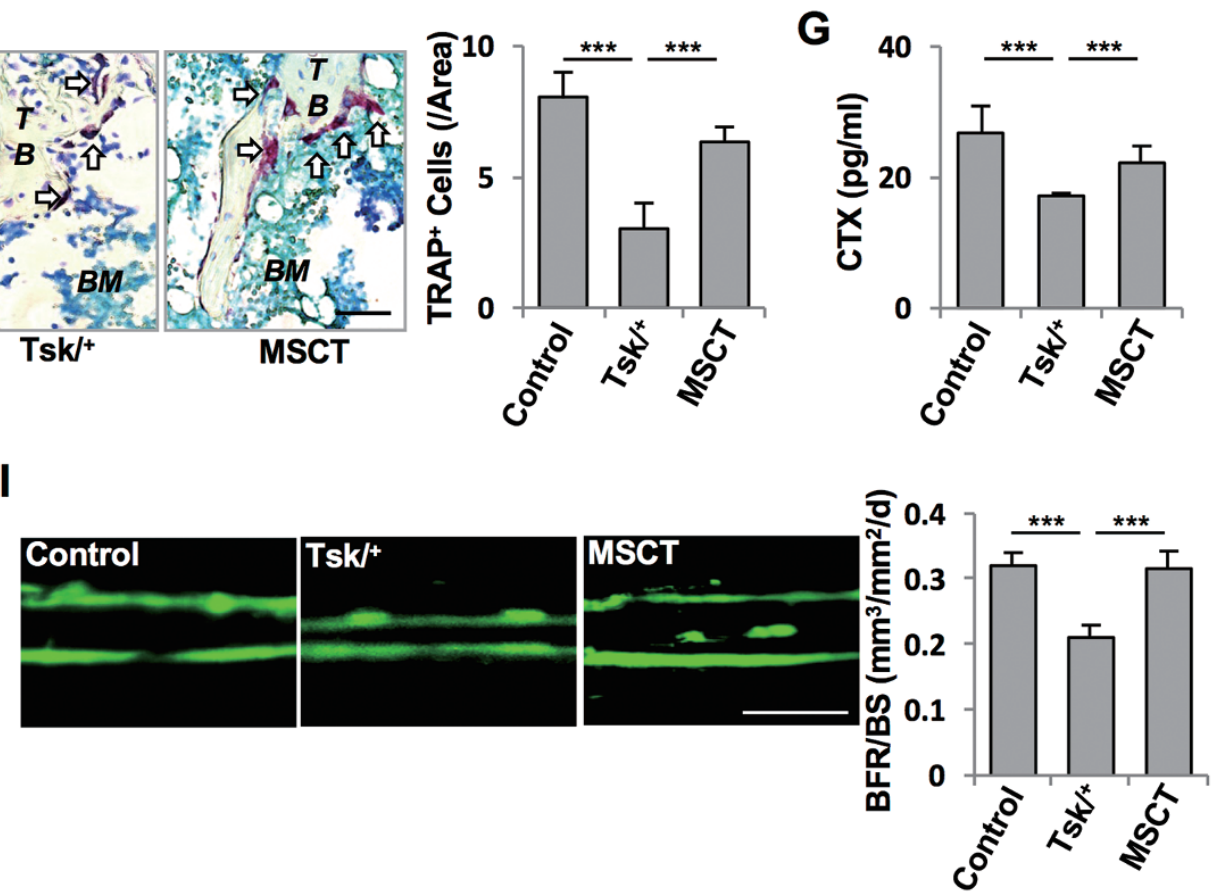

Figure $1 \mathrm{MSCT}$ rescued osteopenia in Tsk $/^{+}$mice. (A) Experimental outline describing the use of MSCT to treat $T s k l^{+}$mice ( $n=6$ per group). (B, C) MicroCT analysis showing images (B) and bone mineral density (BMD) (C) of trabecular bone (TB) area in distal femurs of wild-type (WT) control, Tsk/ ${ }^{+}$, and MSCT-treated Tsk/ mice. (D) H\&E staining showing TB volume (yellow-circled area) in WT, Tsk ${ }^{+}$, and MSCT-treated $T s k l^{+}$mouse distal femurs, Scale bar, 1 mm. (E) Immunohistochemical staining of $\mathrm{ALP}^{+}$osteoblasts in WT, Tskl ${ }^{+}$, and MSCT-treated mice. (F) TRAP staining of osteoclasts in distal femur TB and bone marrow (BM) in WT, Tskl $l^{+}$, and MSCT-treated Tsk/ mice. Arrowheads, TRAP ${ }^{+}$osteoclasts (purple cells). Scale bar, $25 \mu \mathrm{m}$. (G, H) ELISA analysis showing the levels of serum CTX (G) and SRANKL (H) in WT, Tsk ${ }^{+}$, and MSCT-treated Tsk ${ }^{+}$ mice. (I) Calcein double labeling of the metaphyseal TB in the distal femurs showing the bone turnover rate in WT, Tsk ${ }^{+}$, and MSCT-treated $T s k l^{+}$mice. Scale bar, $25 \mu \mathrm{m}$. All experimental data were verified in at least three independent experiments. Error bars represent the s.d. from the mean values. ${ }^{* * *} P<0.005 ;{ }^{* *} P<0.001 ;{ }^{*} P<0.05$. 
A

D

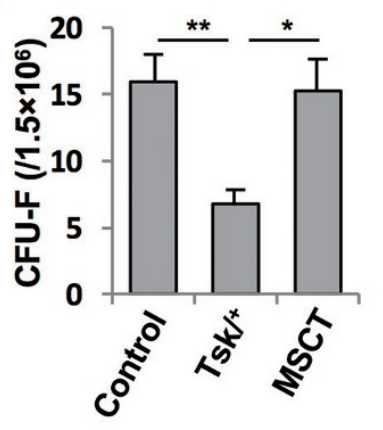

B

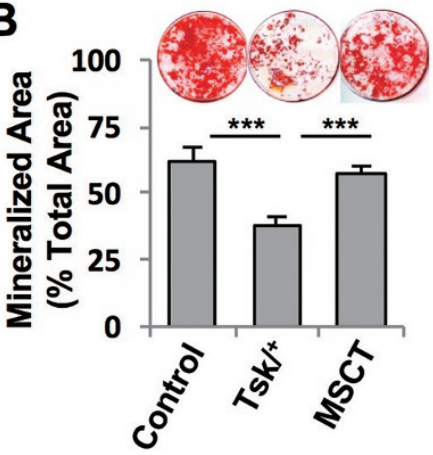

C
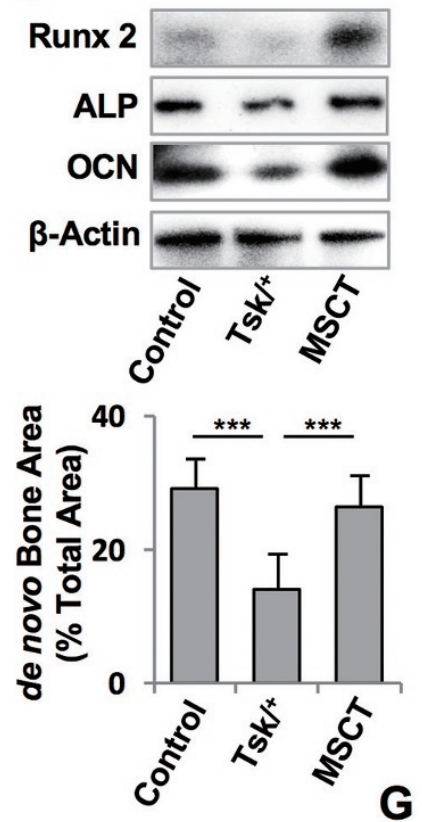

E

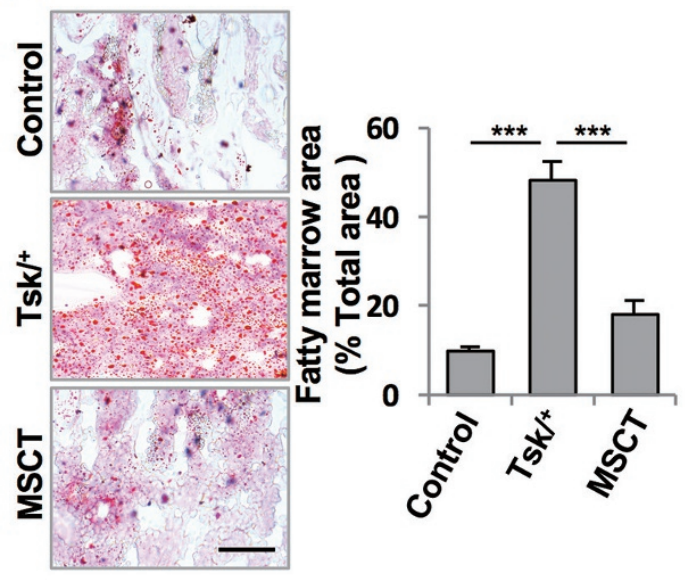

$\mathbf{F}$

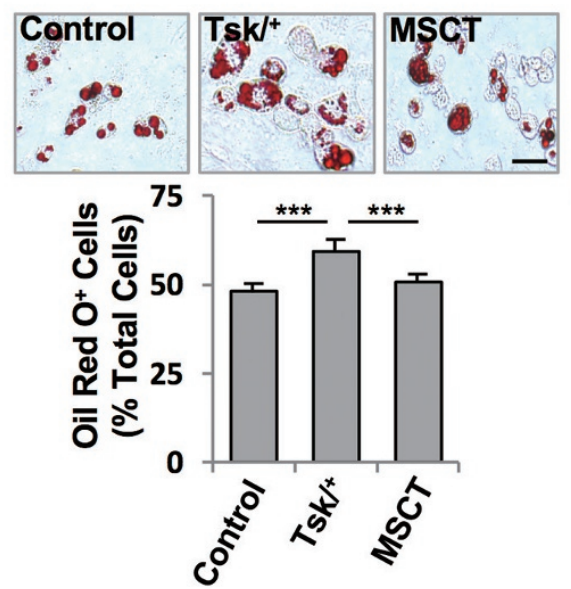

LPL

PPARY

B-Actin

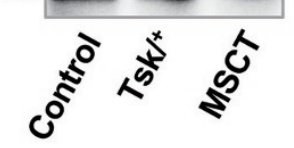

Figure 2 MSCT rescued impaired lineage differentiation of BMMSCs in $T s k{ }^{+}$mice. (A) Toluidine blue staining showing the number of CFU-F from WT control, Tsk/ ${ }^{+}$, and MSCT-treated Tsk/ ${ }^{+}$BMMSCs. (B) Alizarin red staining of WT, $T s k l^{+}$, and MSCT-treated Tsk ${ }^{+}$BMMSCs showing the capacity to form mineralized nodules when cultured under the osteoinductive conditions. (C) Western blotting analysis of WT, Tsk/ ${ }^{+}$, and MSCT-treated Tsk/ BMMSCs showing the expression levels of the osteogenic genes Runx2, ALP, and OCN. $\beta$-Actin was used as a protein loading control. (D) Subcutaneous implantation of WT, $\mathrm{Tsk}^{+}$, and MSCT-treated Tsk ${ }^{+}$BMMSCs in immunocompromised mice showing that new bone (B) and connective tissue (CT) were generated around the HA/TCP $(\mathrm{HA})$ carrier at 8 weeks post implantation. Scale bar, $50 \mu \mathrm{m}$. A semiquantitative analysis shows the amount of bone formation in BMMSC implants. (E) Histological images of distal femurs showing the number of adipocytes in WT, $T s k l^{+}$, and MSCT-treated $T s k l^{+}$mouse bone marrow, as assessed by Oil red O staining. Scale bar, $50 \mu \mathrm{m}$. (F) The number of Oil red $\mathrm{O}^{+}$cells in WT, Tsk ${ }^{+}$, and MSCT-treated Tsk/ ${ }^{+}$BMMSCs under the adipoinductive conditions. (G) The expression levels of adipogenic genes PPAR $\gamma$ and $L P L$ in WT, Tsk ${ }^{+}$, and MSCT-treated Tsk/ $/^{+}$BMSCs under the adipoinductive conditions. All experimental data were verified in at least three independent experiments. Error bars represent the s.d. from the mean values. ${ }^{* * *} P<0.005 ;{ }^{* *} P<0.001 ;{ }^{*} P<0.05$.

cuing BMMSC lineage differentiation.

MSCT transfers miR-151-5p to Tsk/+-recipient BMMSCs to rescue impaired osteo-/adipo-lineage differentiation
Since $\mathrm{T}_{\mathrm{H}} 2$ cell infiltration and activation of IL4 signaling may contribute to SSc disease phenotypes [9-11], we examined whether MSCT regulates IL4 downstream signaling in $T_{s} k l^{+}$BMMSCs. Western blot analysis 
showed upregulation of IL4 signaling downstream molecules such as phosphoinositide 3-kinase (PI3K)-p110, phosphorylated Akt (p-Akt), and phosphorylated mTOR (p-mTOR) in $T s k /^{+}$BMMSCs, whereas MSCT markedly downregulated the levels of PI3K, p-Akt, and p-mTOR (Figure 3A). Interestingly, the expression level of IL$4 \mathrm{R} \alpha$ was also rescued by MSCT, as shown by western blotting and quantitative PCR (qPCR) experiments
A

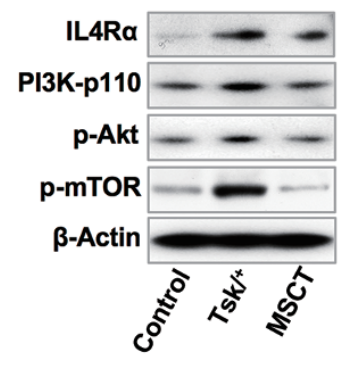

B

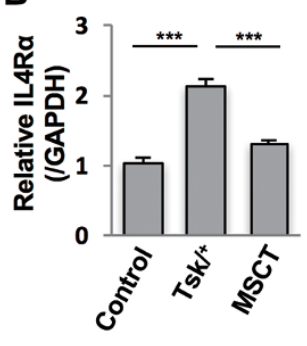

C

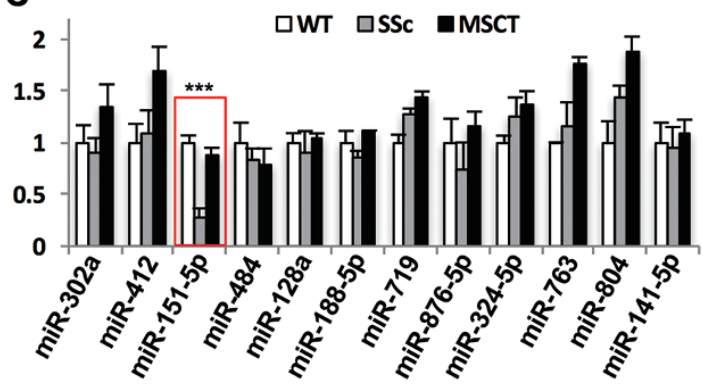

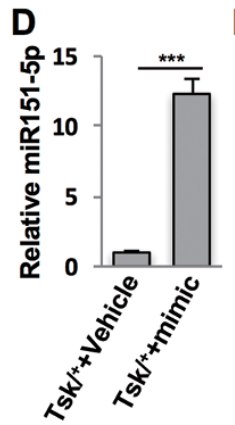

E $\quad F$

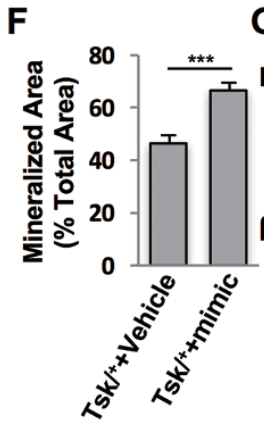

G

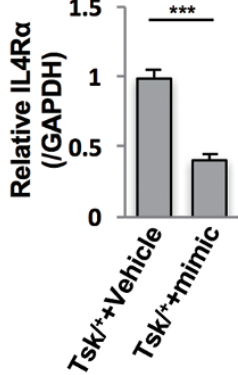

Runx 2

$\mathbf{H}$
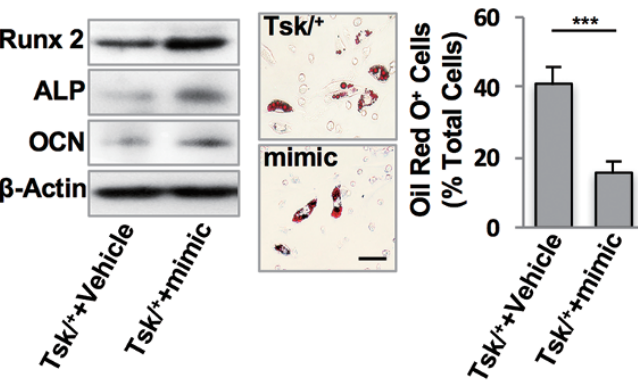

I
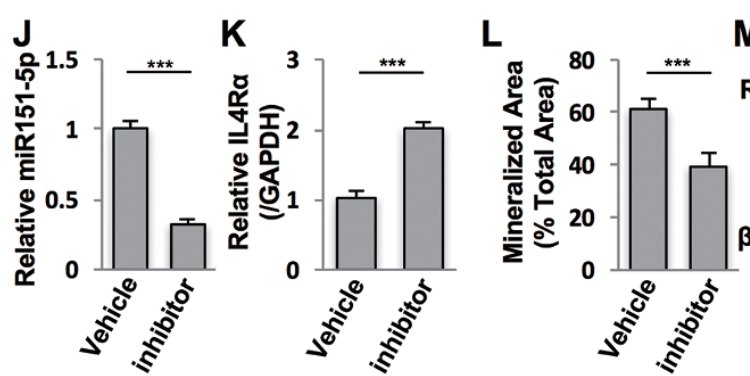

M

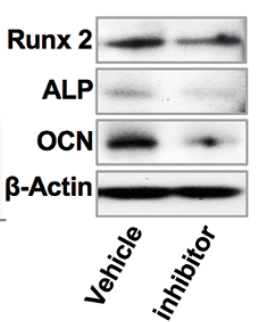

$\mathbf{N}$

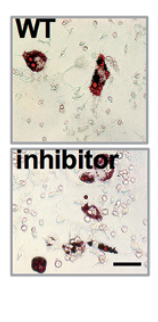

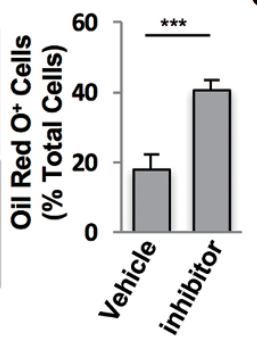

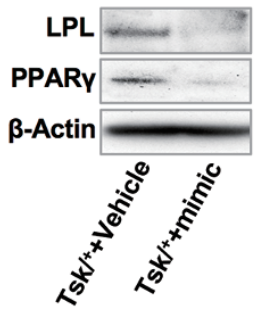

0

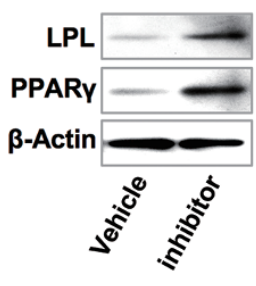

Figure 3 MSCT transferred miR-151-5p into Tsk/ BMMSCs to regulate IL4R $\alpha / m T O R$ signaling. (A) Western blot showing the expression levels of mTOR signaling genes, including $I L 4 R \alpha, P I 3 K-p 110, p-A k t$, and $p-m T O R$ in WT, Tsk ${ }^{+}$, and MSCT-treated $T s k l^{+}$BMMSCs. (B) qPCR analysis showing the expression levels of $\| 4 r \alpha$ in WT, $T s k l^{+}$, and MSCT-treated Tsk ${ }^{+}$BMMSCs. (C) qPCR analysis showing the levels of miRNAs in WT, Tsk ${ }^{+}$, and MSCT-treated $T s k l^{+}$BMMSCs. (D) qPCR analysis showing the levels of miR-151-5p in vehicle and miR-151-5p mimic-treated Tsk/ ${ }^{+}$BMMSCs. (E) qPCR analysis showing the expression levels of $/ / 4 r \alpha$ in vehicle and miR-151-5p mimic-treated $T s k I^{+}$BMMSCs. (F) Alizarin red staining showing that vehicle and miR-151-5p mimic-treated Tsk/ ${ }^{+}$BMMSCs form mineralized nodules when cultured under osteoinductive conditions. (G) Western blotting analysis showing the expression levels of the osteogenic genes Runx2, ALP, and OCN in vehicle- and miR151-5p mimic-treated Tsk/ ${ }^{+}$BMMSCs. $\beta$-Actin was used as a protein loading control. $(\mathbf{H})$ The number of Oil red $\mathrm{O}^{+}$cells in vehicle- and miR-151-5p mimic-treated Tsk ${ }^{+}$BMMSCs under the adipoinductive conditions. (I) Western blotting showing the expression levels of the adipogenic genes PPAR $\gamma$ and $L P L$ in vehicle- and miR-151-5p mimic-treated Tsk ${ }^{+}$BMMSCs when cultured under adipoinductive conditions. (J) qPCR analysis showing the levels of miR-151-5p in vehicle- and miR-151-5p inhibitor-treated WT BMMSCs. (K) qPCR analysis showing the expression levels of //4ra in vehicle- and miR-151-5p inhibitor-treated WT BMMSCs. (L) Alizarin red staining showing that vehicle- and miR-151-5p inhibitor-treated WT BMMSCs form mineralized nodules under osteoinductive conditions. (M) Western blotting showing the expression levels of the osteogenic genes Runx2, ALP, and OCN in vehicle- and miR-151-5p inhibitor-treated WT BMMSCs. $\beta$-Actin was used as a protein loading control. (N) The number of Oil red $\mathrm{O}^{+}$cells in vehicle- and miR-151-5p inhibitor-treated WT BMMSCs under adipoinductive conditions. (O) Western blotting showing the expression levels of the adipogenic genes PPAR $\mathrm{O}$ and $L P L$ in vehicle- and miR151-5p inhibitor-treated WT BMMSCs when cultured under adipoinductive conditions. All experimental data were verified in at least three independent experiments. Error bars represent the s.d. from the mean values. ${ }^{* *} P<0.005$. 
(Figure $3 \mathrm{~A}$ and $3 \mathrm{~B}$ ). This finding prompted us to further examine how MSCT regulates IL4R $\alpha$ expression in $T s k l^{+}$BMMSCs. As MSCs are able to transfer miRNAs to surrounding cells in cancer and other disease models [38-40], here we asked whether miRNAs, which are small non-coding RNAs ( 22 nucleotides) capable of silencing gene expression at post-transcriptional level [41], are involved in MSCT-mediated regulation of IL4R $\alpha$ expression. We used a software available online, MicroCosm Targets, to computationally identify 19 miRNAs that may target Il4ra gene expression (Supplementary information, Table S1). Although the levels of several miRNAs were increased after MSCT treatment in SSc mice, qPCR analysis showed that only miR151-5p was significantly decreased in $T s k /^{+}$BMMSCs (Figure 3C). After MSCT treatment, the level of miR-151-5p was significantly increased in $T s k /^{+}$BMMSCs, suggesting that IL4R $\alpha$ expression may be regulated by miR-151$5 p$ (Figure 3C). To further confirm the functional role of miR-151-5p, we used different amounts of miR151-5p mimic and inhibitor to treat BMMSCs and found that 25 pmol miR151-5p mimic and inhibitor provided optimal effects on regulating IL4R $\alpha$ expression in BMMSCs (Supplementary information, Figure S1). We treated $T s k{ }^{+}$ BMMSCs with a miR-151-5p mimic to increase the levels of miR-151-5p and found that Il4r $\alpha$ expression was significantly decreased (Figure 3D and 3E). Moreover, miR-151-5p mimic improved osteogenic differentiation of $T s k /^{+}$BMMSCs, as indicated by alizarin red staining to show increased mineralized nodule formation and western blotting to show elevated expression of Runx2, ALP, and $O C N$ (Figure $3 \mathrm{~F}$ and $3 \mathrm{G}$ ). Conversely, miR-151$5 \mathrm{p}$ mimic inhibited adipogenic differentiation in $T s k /^{+}$ BMMSCs, as indicated by a decreased number of Oil red O-positive cells and downregulation of PPAR $\gamma$ and $L P L$ (Figure $3 \mathrm{H}$ and $3 \mathrm{I}$ ). In contrast, miR-151-5p inhibitor treatment reduced the levels of miR-151-5p, elevated the expression levels of Il4ra in WT BMMSCs (Figure 3J and $3 \mathrm{~K}$ ), and impaired osteogenic differentiation, as indicated by reduced mineralized nodule formation (Figure $3 \mathrm{~L}$ ), downregulation of Runx2, $A L P$, and $O C N$ (Figure $3 \mathrm{M}$ ), an elevated number of Oil red O-positive cells (Figure $3 \mathrm{~N}$ ), and upregulation of the adipogenic genes $P P A R \gamma$ and $L P L$ (Figure $3 \mathrm{O}$ ).

As miR-151-5p is $100 \%$ identical in mice and humans (Supplementary information, Figure S2A), we reasoned that human miR-151-5p may also target human Il4ro, as predicted by microRNA.org (Supplementary information, Figure S2B). We further confirmed that miR-151$5 p$ inhibitor treatment significantly reduced the levels of miR-151-5p (Supplementary information, Figure S3A), upregulated hIl4ro expression (Supplementary informa- tion, Figure S3B), decreased osteogenic differentiation as indicated by reduced mineralized nodule formation (Supplementary information, Figure S3C), downregulated expression of Runx2, ALP, and $O C N$ (Supplementary information, Figure S3D), elevated the number of Oil red O-positive cells (Supplementary information, Figure S3E), and upregulated expression of the adipogenic genes PPAR $\gamma$ and LPL (Supplementary information, Figure S3F) in human BMMSCs. To confirm that IL4 can induce downstream mTOR signaling in human BMMSCs, we showed that recombinant IL4 treatment resulted in upregulation of $\mathrm{p}$-mTOR with downregulation of Runx2, ALP, and $O C N$ and decreased in vitro mineralized nodule formation (Supplementary information, Figure S4A and S4B). Treatment with rapamycin, an mTOR signaling specific inhibitor, rescued the IL4-induced osteogenic differentiation deficiency (Supplementary information, Figure S4A and S4B). Next, we used miR151-5p mimic to treat IL4-induced human BMMSCs and found increased levels of miR-151-5p and reduced expression levels of Il4r (Supplementary information, Figure S4C and S4D). In addition, we showed that osteogenic differentiation of IL4-induced human BMMSCs was significantly improved after miR-151-5p mimic treatment, as indicated by alizarin red staining to show increased mineralized nodule formation and western blotting to show elevated expression of Runx2, ALP, and $O C N$ (Supplementary information, Figure S4E and S4F). Conversely, the elevated adipogenic differentiation in IL4-induced human BMMSCs was significantly reduced in the miR151-5p mimic treatment group, as indicated by a decreased number of Oil red O-positive cells and downregulation of PPAR $\gamma$ and LPL (Supplementary information, Figure S4G and S4H). Collectively, these data suggest that MSCT transferred miR-151-5p to $T s k l^{+}$-recipient BMMSCs to block the IL4R $\alpha$ pathway and rescue impaired lineage differentiation of recipient cells.

\section{MSCT employs extracellular vesicles to rescue $T s k{ }^{+}$BMMSCs}

To determine how MSCT transfers miR-151-5p to recipient cells, we co-cultured WT BMMSCs with $T s k{ }^{\dagger}$ BMMSCs in a transwell system to examine whether miR-151-5p can be transferred in vitro. After 3 days of co-culture, we observed elevated intracellular levels of miR-151-5p, decreased Il4ra gene expression, and improved osteogenic differentiation in $T s k /^{+}$BMMSCs, as assessed by $\mathrm{qPCR}$, alizarin red staining, and western blotting experiments, respectively (Figure 4A-4C). These data suggest that WT BMMSCs may release miR-151$5 \mathrm{p}$ to regulate $T s k /^{+}$BMMSCs. As EVs, which include exosomes [42], have been shown to carry RNAs for intercellular crosstalk [31-33], we next examined whether 
A

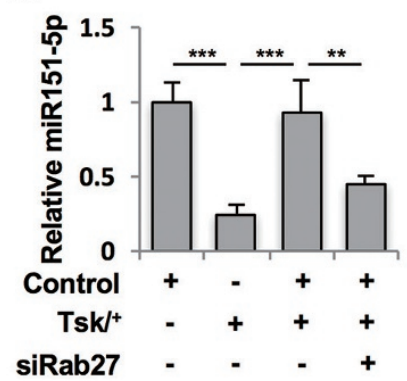

B

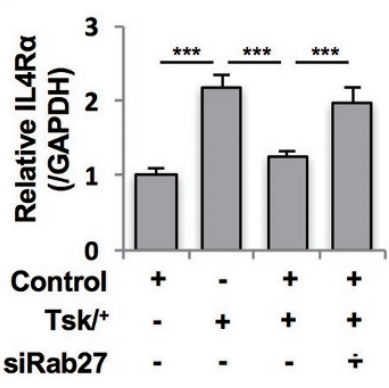

C

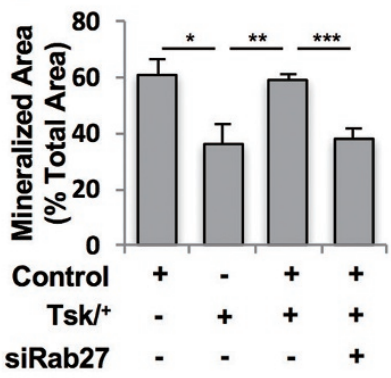

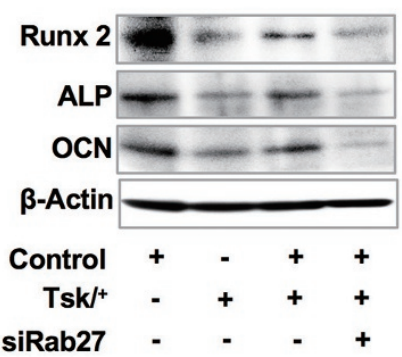

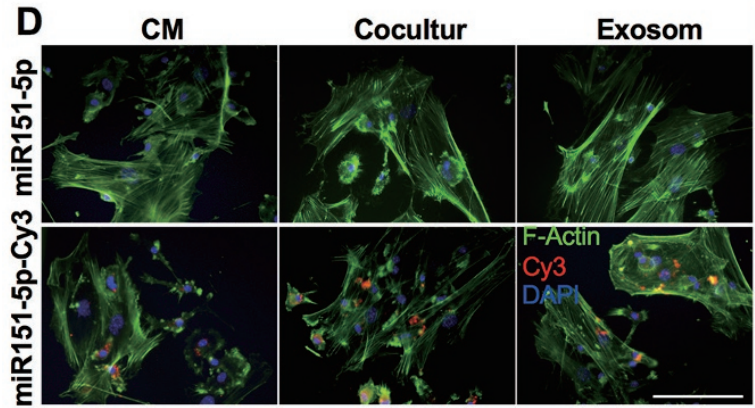

G

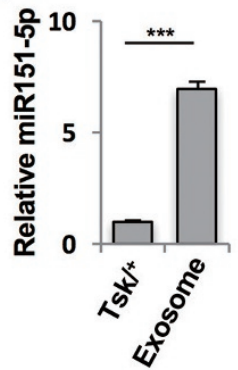

H

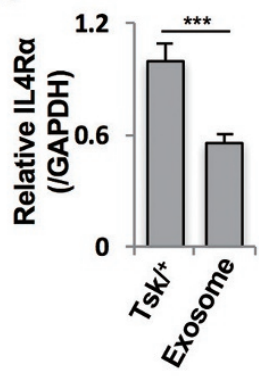

E

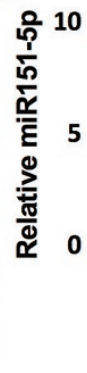

I

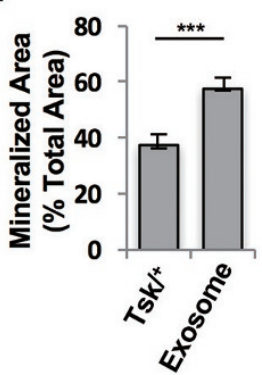

F

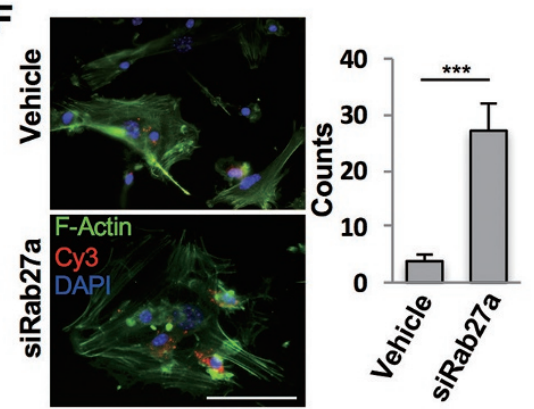

J

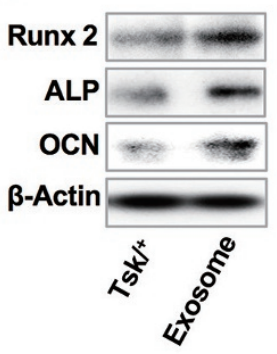

K

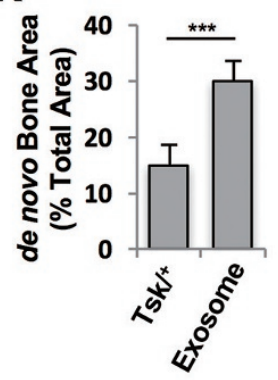

Figure 4 Exosome-mediated miR-151-5p transfer rescued impaired Tsk/ ${ }^{+}$BMMSCs. (A) When Tsk/ ${ }^{+}$BMMSCs were cocultured with WT BMMSCs using a transwell system, qPCR analysis showed that intracellular levels of miR-151-5p in Tsk/ BMMSCs were elevated. Blockage of exosome release from WT BMMSCs by rab27a siRNA attenuated intracellular miR-

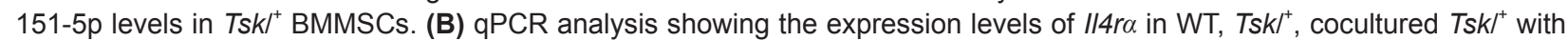
WT, and cocultured Tsk/ ${ }^{+}$with rab27a siRNA-treated WT BMMSCs. (C) Alizarin red staining showing the capacity to form mineralized nodules and western blotting showing the expression levels of osteogenic genes Runx2, ALP, and OCN in WT, $T s k l^{+}$, cocultured $T s k l^{+}$with WT, and cocultured $T s k l^{+}$with rab27a siRNA-treated WT BMMSCs under osteoinductive conditions. $\beta$-Actin was used as a protein loading control. (D) Left panel: after miR-151-5p-Cy3 was transfected into WT BMMSCs, conditioned medium (CM) was harvested and loaded onto Tsk/ $/^{+}$BMMSCs to show that miR-151-5p-Cy3 was able to transfer to Tsk/ ${ }^{+}$BMMSCs. Middle panel: using a transwell co-culture system, miR-151-5p-Cy3-transfected WT BMMSCs were co-cultured with Tsk/ ${ }^{+}$BMMSCs to show that miR-151-5p-Cy3 was able to be transferred from WT to Tsk/ ${ }^{+}$BMMSCs. Right panel: after miR-151-5p-Cy3 was transfected into WT BMMSCs, exosomes were isolated and loaded onto Tsk ${ }^{+}$BMMSCs to show that miR-151-5p-Cy3 was able to transfer via exosomes to Tsk/ ${ }^{+}$BMMSCs. Scale bar, $25 \mu \mathrm{m}$. (E) After miR-151-5pCy3 transfection in WT BMMSCs, miR-151-5p intracellular levels were examined by performing qPCR analysis of transfected CM-treated Tsk/+, cocultured transfected BMMSCs with $T s k l^{+}$, and exosome-treated Tsk/ BMMSCs. (F) To examine the role of Rab27a in exosome-mediated miR-151-5p transfer, Rab27a siRNA was co-transfected with miR151-5p-Cy3 into WT BMMSCs to show that miR-151-5p-Cy3 accumulated in the transfected BMMSCs compared to the scrambled siRNA transfected group, indicating that exosome-mediated miR-151-5p transfer was blocked by Rab27a. Scale bar, $25 \mu \mathrm{m}$. (G-K) After exosomes derived from WT BMMSCs were used to treat $T s k{ }^{+}$BMMSCs, qPCR analysis showed elevated intracellular levels of miR-151-5p (G) and reduced expression levels of $/ / 4 r \alpha(H)$, along with elevated mineralized nodule formation, as assessed by alizarin red staining (I), and increased expression of the osteogenic genes Runx2, ALP, and OCN, assessed by western blotting (J). When implanted into immunocompromised mice subcutaneously, increased new bone formation was observed (K). All experimental data were verified in at least three independent experiments. Error bars represent the s.d. from the mean values. ${ }^{* *} P<0.005 ;{ }^{* *} \mathrm{P}<0.001 ;{ }^{*} \mathrm{P}<0.05$. 
the secretion of EVs by BMMSCs can rescue impaired $T_{s k} /^{+}$BMMSCs. Using an siRNA approach to knockdown rab27a expression (Supplementary information, Figure S5A) to block extracellular vesicle (EV)/exosome secretion [43], we found that rab27a knockdown attenuated WT BMMSC-mediated rescue of intracellular miR151-5p levels and Il4ra expression, as well as osteogenic differentiation in $T_{s k} /^{+}$BMMSCs (Figure 4A-4C). Next, we confirmed that purified EVs/exosomes from BMMSCs expressed exosome-specific markers CD63 and CD81 by western blotting (Supplementary information, Figure S5B) and then we labeled miR-151-5p with $\mathrm{Cy} 3$ red fluorescent dye to show its co-localization with the exosome marker CD63 in BMMSCs (Supplementary information, Figure S5C). In order to examine whether miR-151-5p can transfer between cultured BMMSCs, we co-cultured $T s k /^{+}$BMMSCs with miR151-5p-cy3transfected WT BMMSCs, conditioned medium from transfected BMMSCs, and EVs/exosomes from transfected BMMSCs. We showed that miR-151-5p can be transferred through EVs to $T s k{ }^{+}$BMMSCs (Figure 4D) along with elevated levels of intracellular miR151-5p (Figure 4E). To further confirm EV/exosome-mediated miR-151-5p transfer, we used rab27a siRNA to block EV/exosome release in WT BMMSCs and found that miR151-5p transfer was markedly inhibited (Figure 4F). When EVs from WT BMMSCs were added to cultured $T_{s} k /^{+}$BMMSCs, the levels of intracellular miR-151-5p were increased, and the expression levels of $I l 4 r \alpha$ were decreased (Figure 4G and $4 \mathrm{H}$ ). In addition, the EV treatment was able to improve osteogenic differentiation of $T s k /^{+}$BMMSCs, as assessed by increased mineralized nodule formation, upregulation of the Runx2, ALP, and $O C N$, and increased in vivo bone formation (Figure 4I$4 \mathrm{~K})$. To address whether MSCT releases EVs/exosomes in $T s k{ }^{+}$mice, we transfected the CD63-GFP reporter vector into BMMSCs and performed MSCT in $T s k{ }^{+}$mice. After MSCT, immunofluorescence (IF) staining showed that CD63 co-localized with the BMMSC marker CD105 in both the femur and skin in $T s k /^{+}$mice (Supplementary information, Figure S5D). We next labeled BMMSCs with red fluorescent dye, followed by transfection of the CD63-GFP reporter vector and performed MSCT in Tsk ${ }^{+}$ mice to show that transplanted BMMSCs were not engrafted into the femur or skin in $\left.T_{s} k\right)^{+}$mice (Supplementary information, Figure S5E). To assess whether EVs/ exosomes transfer miR151-5p in vivo, we co-transfected the CD63-GFP reporter vector with Cy3-labeled miR151$5 \mathrm{p}$ into BMMSCs and performed MSCT in $T s k /^{+}$mice. The data showed that MSCT-released CD63-GFP ${ }^{+}$EVs were co-localized with Cy3-miR151-5p, indicating that MSCT was associated with the secretion of exosomal
miR151-5p in $T s k /^{+}$mice (Supplementary information, Figure S5F). Taken together, these experimental data indicate that MSCT-released EVs transferred miR151-5p to $T s k /^{+}$BMMSCs to rescue the disease phenotypes.

We next infused EVs into $T s k{ }^{+}$mice to observe their in vivo therapeutic effects. After intravenous infusion into $T s k{ }^{+}$mice, contents of EVs were detected in bone marrow cells at $24 \mathrm{~h}$ post transplantation (Supplementary information, Figure S5G) and $T s k /^{+}$BMMSCs showed elevated intracellular levels of miR-151-5p (Figure 5A) and reduced expression levels of Il4ra (Figure 5B). To further confirm that the increased miR151-5p was mainly transferred by EVs, we used miR151-5p inhibitor to generate miR-151-5p-depleted EVs and infused them into $T s k /^{+}$mice, and found that miR-151-5p-depleted EVs failed to elevate intracellular levels of miR-151-5p (Figure 5A) and reduce expression levels of Il4ra (Figure 5B). Increased osteogenic differentiation was also observed upon infusion of EVs, as assessed by alizarin red staining to show elevated mineralized nodule formation (Figure 5C), western blotting to show upregulation of the osteogenic genes Runx2, ALP, and $O C N$ (Figure 5D), and in vivo implantation to show elevated bone formation capacity (Figure 5E), while miR-151-5p-depleted EVs failed to increase osteogenic differentiation (Figure $5 \mathrm{C}-5 \mathrm{E}$ ). In contrast, BMMSCs from EV-treated, but not miR-151-5p-depleted EV-treated $T_{s} k /^{+}$mice, showed a significant reduction in adipogenic differentiation, as shown by decreased numbers of Oil red O-positive cells and downregulation of the adipogenic genes $P P A R \gamma$ and $L P L$ (Figure 5F and 5G). MicroCT and histological analysis showed that EV-treated $T s k{ }^{+}$mice have significantly increased BMD and trabecular bone volume compared to untreated $T_{s} k /^{+}$mice (Figure $5 \mathrm{H}$ and $5 \mathrm{I}$ ), whereas miR-151-5p-depleted EVs failed to increase $\mathrm{BMD}$ and trabecular bone volume (Figure $5 \mathrm{H}$ and $5 \mathrm{I}$ ). The numbers of adipocytes in EV/exosome-treated, but not miR-151-5p-depleted EV-treated $T s k /^{+}$mouse bone marrow were significantly reduced when compared to untreated $\left.T s k\right|^{+}$mice, as assessed by Oil red $\mathrm{O}$ staining (Figure 5J). These data indicate that EVs/exosomes play an important role in the regulation of miR-151-5p levels to rescue $T s k /^{+}$BMMSC function and osteopenia. To further confirm MSCT releasing EVs/exosomes to rescue $\left.T s k\right|^{+}$phenotypes, we blocked EV/exosome secretion in vivo by treating BMMSCs with rab27a siRNA and performed MSCT in $T s k l^{+}$mice. The therapeutic effects of sirab27a-treated BMMSCs were significantly decreased when compared to the control MSCT group, as assessed by microCT and histological analysis to show BMD and trabecular bone volume (Supplementary information, Figure S6A and S6B) and Oil red O staining to show 
A

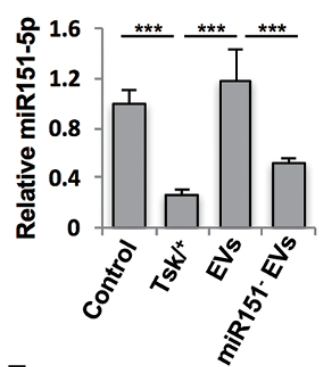

$\mathbf{F}$

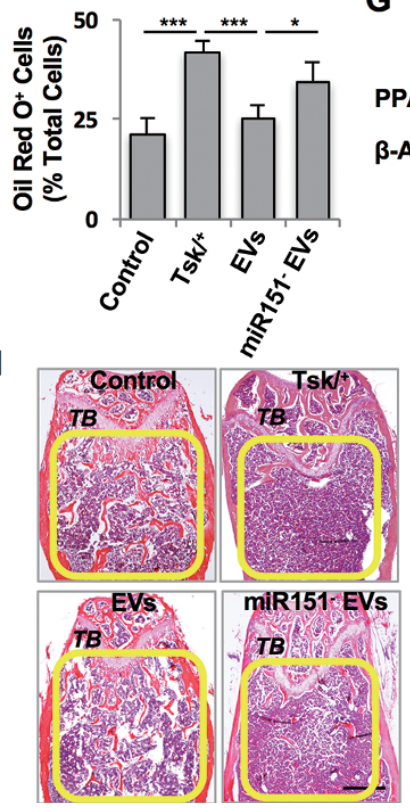

C

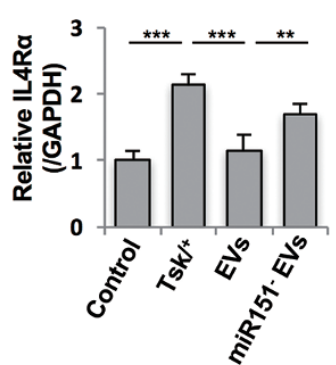

G
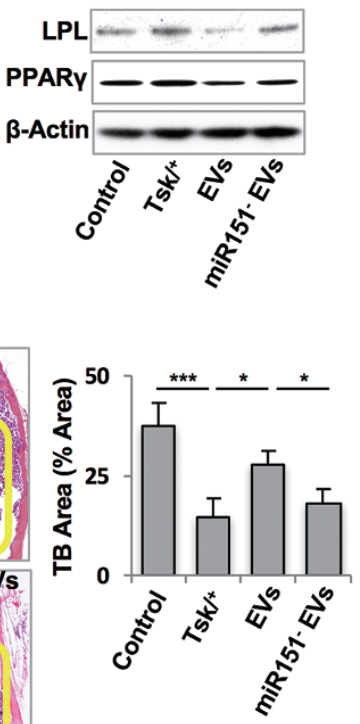

H
D

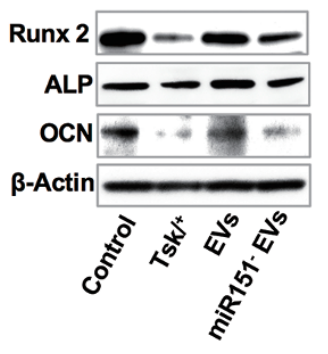

E

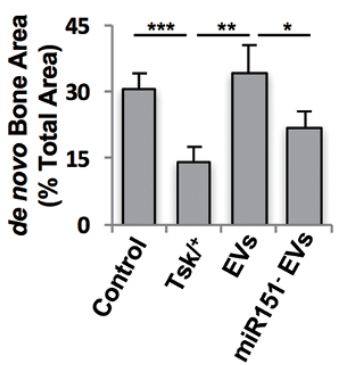

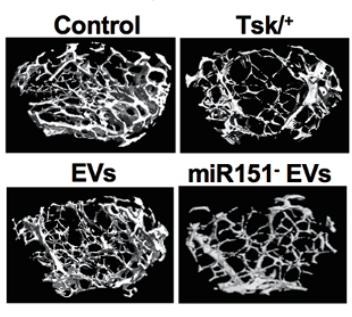

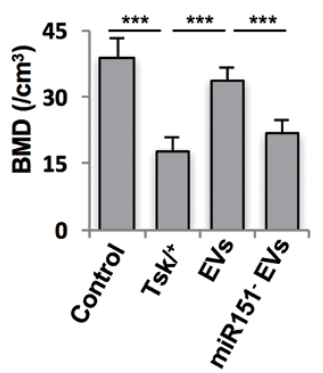

J
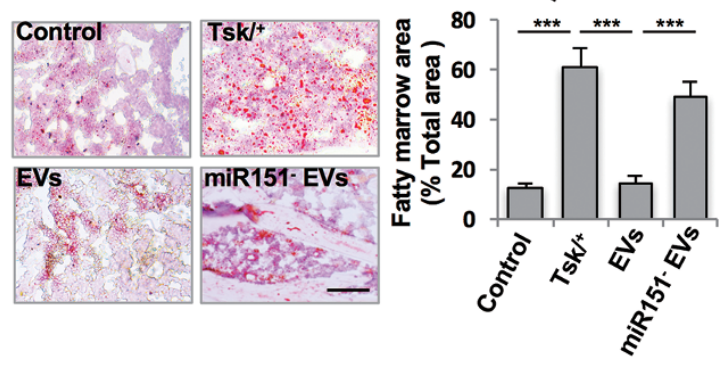

Figure 5 Systemic infusion of exosomes rescued osteopenia in $T s k{ }^{+}$mice through miR151-5p transfer. (A) qPCR analysis showing the levels of miR-151-5p in WT, $T s k l^{+}$, exosome-treated $T s k l^{+}$, and miR-151-5p-depleted exosome-treated $T s k l^{+}$ BMMSCs. (B) qPCR analysis showing the expression levels of $\| / 4 r \alpha$ in WT, Tsk $\left.\right|^{+}$, exosome-treated $T s k I^{+}$, and miR-151-5pdepleted exosome-treated Tsk/ $/^{+}$BMMSCs. (C) Alizarin red staining showing that WT, $T s k l^{+}$, exosome-treated $T s k l^{+}$, and miR151-5p-depleted exosome-treated Tsk ${ }^{+}$BMMSCs form mineralized nodules under osteoinductive conditions. (D) Western blotting analysis showing the expression levels of the osteogenic genes Runx2, ALP, and OCN in WT, $T s k t^{+}$, exosome-treated $T s k l^{+}$, and miR-151-5p-depleted exosome-treated Tsk/ ${ }^{+}$BMMSCs. $\beta$-Actin was used as a protein loading control. (E) When WT, $T s k l^{+}$, exosome-treated $T s k l^{+}$, and miR-151-5p-depleted exosome-treated $T s k l^{+}$BMMSCs were implanted into immunocompromised mice subcutaneously, newly formed bone was observed. (F) The number of Oil red $\mathrm{O}^{+}$cells observed in WT, $T s k l^{+}$, exosome-treated $T s k l^{+}$, and miR-151-5p-depleted exosome-treated Tsk $I^{+}$BMMSCs cultured under adipoinductive conditions. (G) Western blotting showing the expression levels of adipogenic genes PPAR $\gamma$ and $L P L$ in WT, $T s k /^{+}$, exosome-treated $T s k l^{+}$, and miR-151-5p-depleted exosome-treated $T s k l^{+}$BMMSCs cultured under adipoinductive conditions. (H) MicroCT analysis showing BMD and TB area in distal femurs of $W T, T s k /^{+}$, exosome-treated $T s k l^{+}$, and miR-151-5p-depleted exosome-treated $T s k l^{+}$mice. (I) H\&E staining showing TB volume (yellow-circled area) in WT, $T s k l^{+}$, exosome-treated $T s k /^{+}$, and miR-151-5p-depleted exosome-treated $T s k /^{+}$mouse distal femurs. Scale bar, $1 \mathrm{~mm}$. (J) Histological images of distal femurs showing the number of adipocytes in WT, $T s k l^{+}$, exosome-treated $T s k l^{+}$, and miR-151-5p-depleted exosome-treated $T s k l^{+}$ mouse bone marrow based on Oil red $O$ staining. Scale bar, $50 \mu \mathrm{m}$. All experimental data were verified in at least three independent experiments. Error bars represent the s.d. from the mean values. ${ }^{* \star *} P<0.005 ;{ }^{* \star} P<0.001 ;{ }^{*} P<0.05$.

adipocytes in $T s k /^{+}$mouse bone marrow (Supplementary information, Figure S6C).

Overexpression of miR-151-5p rescues disease pheno- types in $\mathrm{Tsk}^{+}$mice

In light of miR-151-5p negatively regulating $I L 4 R \alpha$ expression in $T s k /^{+}$BMMSCs, we next assessed the effects of overexpressing miR-151 in $T s k /^{+}$mice by ad- 
enoviral vector with a green fluorescent protein (GFP) reporter (Ad-miR-151). Ad-miR151 $\left(2 \times 10^{9}\right.$ p.f.u.) or an empty vector (Ad-GFP) was injected into $T s k{ }^{+}$mice three times a week for up to 4 weeks (Figure 6A). Forty-eight hours after the last injection, we used immunofluorescent analysis to show GFP expression in Ad-
miR-151-treated $T s k /^{+}$bone marrow cells (Figure 6B). Ad-miR-151-treated $T_{s} k /^{+}$BMMSCs were collected to show elevation of the intracellular levels of miR-151-5p (Figure 6C) and reduced expression levels of Il4ro (Figure 6D). Osteogenic differentiation of BMMSCs from Ad-miR-151-treated $T s k /^{+}$mice was markedly improved
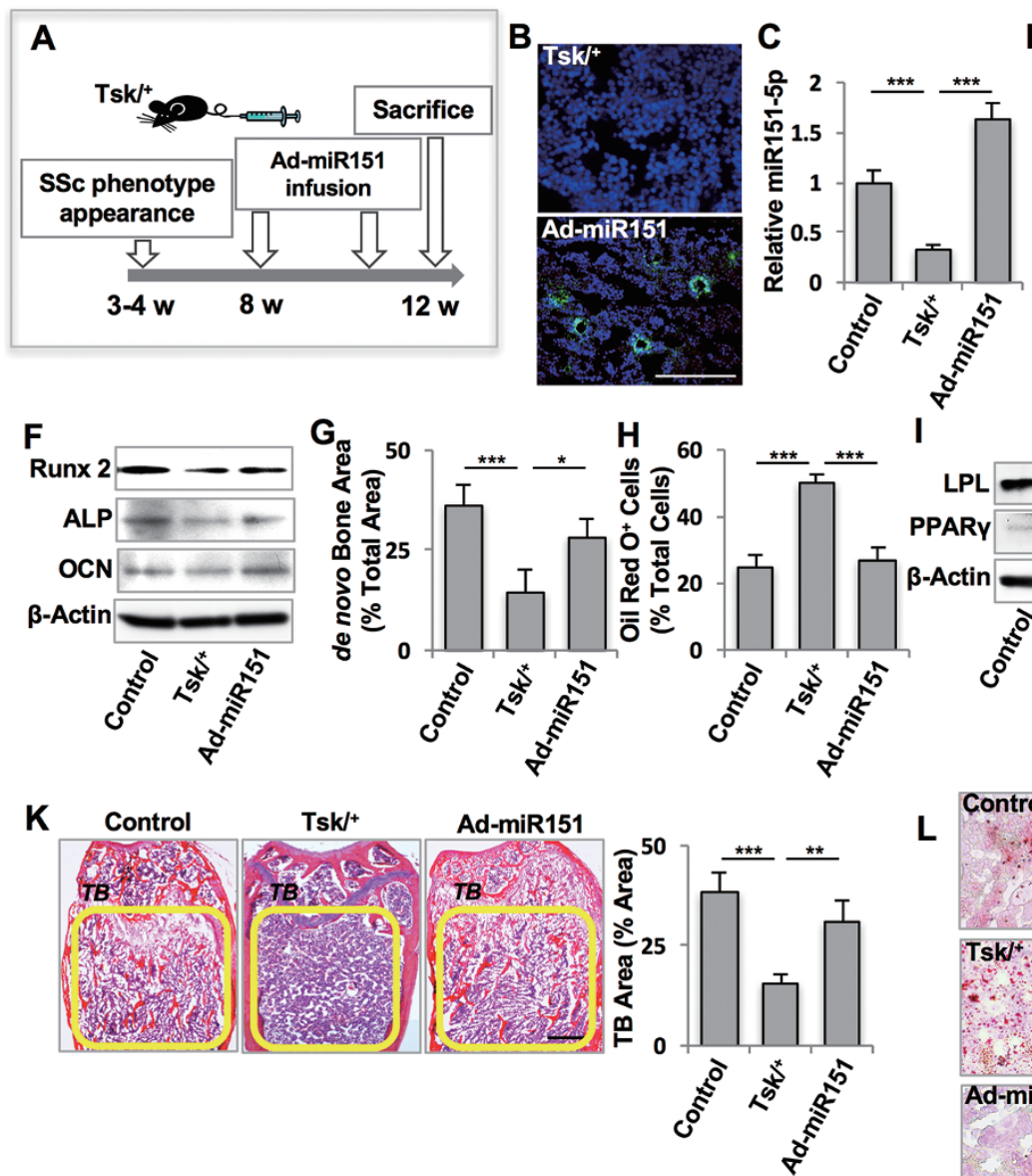

D

E
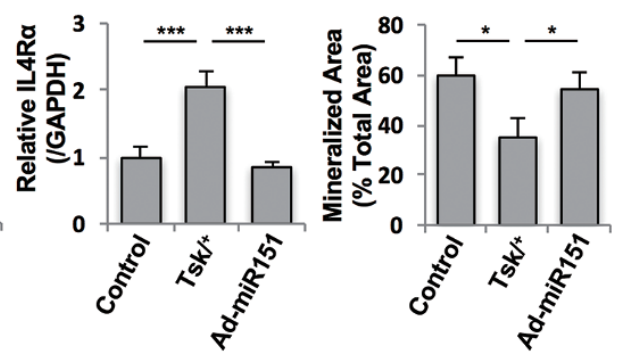

1
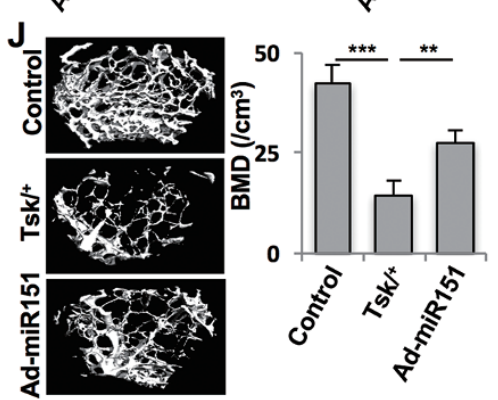

$\mathbf{L}$

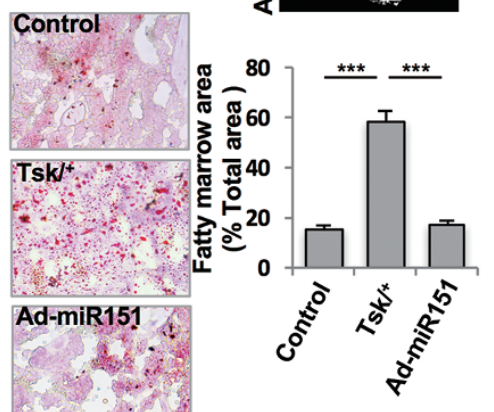

Figure 6 Adenovirus-mediated miR-151-5p overexpression rescued impaired Tsk/ $/^{+}$BMMSCs. (A) Experimental outline describing the use of Ad-miR-151 to treat $T s k t^{+}$mice ( $n=6$ per group). (B) Ad-miR-151-GFP was observed in bone marrow cells of Tskl ${ }^{+}$mice. Scale bar, $25 \mu \mathrm{m}$. (C) qPCR analysis showing the levels of miR-151-5p in WT, Ad-GFP, and Ad-miR-151treated Tsk ${ }^{+}$BMMSCs. (D) qPCR analysis showing the expression levels of $\| 4 r \alpha$ in WT, Ad-GFP, and Ad-miR-151-treated $T_{s k} l^{+}$BMMSCs. (E) Alizarin red staining showing mineralized nodule formation in WT, Ad-GFP, and Ad-miR-151-treated Tsk ${ }^{+}$ BMMSCs cultured under osteoinductive conditions. (F) Western blotting showing the expression levels of the osteogenic genes Runx2, ALP, and OCN in WT, Ad-GFP, and Ad-miR-151-treated Tsk $I^{+}$BMMSCs. $\beta$-Actin was used as a protein loading control. (G) When WT, Ad-GFP, and Ad-miR-151-treated Tsk/ ${ }^{+}$BMMSCs were subcutaneously implanted into immunocompromised mice, newly formed bone was observed. (H) The number of Oil red $\mathrm{O}^{+}$cells in WT, Ad-GFP, and Ad-miR-151treated Tsk ${ }^{+}$BMMSCs under the adipoinductive conditions. (I) Western blotting showing the expression levels of adipogenic genes PPAR $\gamma$ and LPL in WT, Ad-GFP, and Ad-miR-151-treated Tsk/ BMMSCs cultured under adipoinductive conditions. (J) MicroCT analysis showing BMD and TB area in distal femurs of WT, Ad-GFP, and Ad-miR-151-treated Tsk ${ }^{+}$mice. (K) H\&E staining showing TB volume (yellow-circled area) in WT, Ad-GFP, and Ad-miR-151-treated mouse distal femurs. Scale bar, $1 \mathrm{~mm}$. (L) Histological images of distal femurs showing the number of adipocytes in WT, Ad-GFP, and Ad-miR-151-treated mouse bone marrow, assessed by Oil red $O$ staining. Scale bar, $50 \mu \mathrm{m}$. All experimental data were verified in at least three independent experiments. Error bars represent the s.d. from the mean values. ${ }^{* *} P<0.005 ;{ }^{* \star} P<0.001 ;{ }^{*} P<0.05$. 
when compared to the Ad-GFP-treated $T s k{ }^{+}$BMMSCs, as analyzed by alizarin red staining to show elevated mineralized nodule formation (Figure 6E), western blotting to show increased expression of the osteogenic genes Runx2, $A L P$, and $O C N$ (Figure 6F), and in vivo BMMSC implantation to show elevated new bone formation (Figure 6G). In contrast, BMMSCs isolated from Ad-miR-151-treated $T s k /^{+}$mice showed significantly less adipogenic differentiation than BMMSCs from Ad-GFPtreated $T s k /^{+}$mice, as shown by a decreased number of Oil red O-positive cells and downregulation of PPAR $\gamma$ and $L P L$ (Figure $6 \mathrm{H}$ and $6 \mathrm{I}$ ). Histological and microCT analysis showed that Ad-miR-151 treatment elevated BMD and trabecular bone volume compared to Ad-GFPtreated $T s k{ }^{+}$mice (Figure $6 \mathrm{~J}$ and $6 \mathrm{~K}$ ). The number of adipocytes in the bone marrow of Ad-miR-151-treated $T s k I^{+}$ mice was significantly reduced compared to Ad-GFPtreated $T s k{ }^{+}$mice, as assessed by Oil red O staining (Figure 6L). To examine whether overexpressing miR151$5 p$ can increase therapeutic effects of EVs, we treated BMMSCs with Ad-miR151 to generate miR151-overexpressed (miR151-OE) EVs and subsequently infused these EVs into $T s k l^{+}$mice. Treatment with EVs from $0.2 \times 10^{6}$ BMMSCs is considered a standard dosage to generate a therapeutic response. Therefore, we infused $10 \%$ of this standard dose of EVs (i.e., EVs from $0.02 \times$ $10^{6}$ BMMSCs) from miR151-5p-overexpressed BMMSCs or control WT BMMSCs into $T s k /^{+}$mice to examine whether miR151-5p overexpression in BMMSCs could elevate the therapeutic effects of EVs. The results showed that this small dose of EVs from miR151-overexpressed BMMSCs, but not from WT BMMSCs, had similar therapeutic effects to those observed in EVs from the $0.2 \times 10^{6}$ BMMSCs group, as assessed by microCT and histological analysis (Supplementary information, Figure S7A and S7B) and Oil red O staining (Supplementary information, Figure S7C).

Next, we examined whether systemic infusion of AdmiR-151 rescues autoimmune and connective tissue disorders in $T s k I^{+}$mice, which represent major phenotypes of SSc. To evaluate skin fibrosis, we found that skin hypodermal thickness was reduced to a level equivalent to the control group after systemic administration of Ad-miR-151 (Figure 7A). $T s k /^{+}$mice showed increased levels of antinuclear antibody (ANA), anti-double strand DNA (dsDNA) IgG and IgM antibodies, creatinine in serum, and urine proteins (Figure 7B-7F). When compared to the Ad-GFP treatment group, Ad-miR-151 treatment significantly reduced the levels of ANA, dsDNA IgG and $\operatorname{IgM}$, serum creatinine, and urine protein in $T s k l^{+}$ mice (Figure 7B-7F). As mature miR-151-5p is able to directly target Il4ro, a key component of $\mathrm{T}_{\mathrm{H}} 2$ cytokine signaling in the regulation of fibrotic tissue deposition and bone homeostasis in SSc [9-11], we hypothesized that systemic Ad-miR-151 infusion downregulates $\mathrm{T}_{\mathrm{H}} 2$ differentiation in $T_{s} k /^{+}$mice. To evaluate the efficiency of in vivo Ad-miR-151 treatment, serum levels of miR-151$5 \mathrm{p}$ were examined by qPCR and we found a markedly decreased level of miR-151-5p in $T s k{ }^{+}$mice compared to control littermates (Supplementary information, Figure S8A). Ad-miR-151 treatment significantly elevated serum levels of miR-151-5p (Supplementary information, Figure S8A). Flow cytometric analysis showed that $T s k I^{+}$mice have significantly increased levels of $\mathrm{T}_{\mathrm{H}} 2$ cells (Supplementary information, Figure S8B) and $\mathrm{T}_{\mathrm{H}} 17$ cells (Supplementary information, Figure S9A), reduced levels of regulatory $\mathrm{T}$ cells (Tregs), and unaltered levels of $\mathrm{T}_{\mathrm{H}} 1$ cells compared with control littermates (Supplementary information, Figure S9B and S9C). Ad-miR-151 treatment markedly reduced the levels of $\mathrm{T}_{\mathrm{H}} 2$ cells (Supplementary information, Figure S8B), but not $\mathrm{T}_{\mathrm{H}} 17$ cells, $\mathrm{T}_{\mathrm{H}} 1$ cells, or Tregs (Supplementary information, Figure S9A-S9C). ELISA confirmed that Ad-miR-151 treatment significantly reduced serum levels of IL4 in $T s k{ }^{+}$mice (Supplementary information, Figure S8C). Moreover, we found that Ad-miR-151 treatment significantly reduced $\mathrm{CD}^{+} \mathrm{IL}^{+} \mathrm{T}_{\mathrm{H}} 2$ cell infiltration, a major pathological phenotype in $T s k /^{+}$mice [9-11], as confirmed by IF staining (Supplementary information, Figure S9D). To further examine the relationship between miR-151-5p and reduced $\mathrm{T}_{\mathrm{H}} 2$ cell differentiation, we infected naive $T s k /^{+} \mathrm{CD} 4 \mathrm{~T}$ cells with Ad-miR-151 to assess the effects on $\mathrm{T}$ helper cell differentiation, activated under either non-polarized $\mathrm{T}_{\mathrm{H}} 0$ or $\mathrm{T}_{\mathrm{H}} 2$ conditions. Ad-miR- 151 treatment promoted a marked increase in miR-151-5p in differentiated $\mathrm{T}$ cells under $\mathrm{T}_{\mathrm{H}} 0$ or $\mathrm{T}_{\mathrm{H}} 2$ conditions (Supplementary information, Figure S8D). In the presence of increased miR-151$5 \mathrm{p}$, we observed downregulation of the $\mathrm{T}_{\mathrm{H}} 2$ genes, Il4 and Gata3, compared to T cells infected with control AdGFP (Supplementary information, Figure S8E). ELISA further confirmed that Ad-miR-151 treatment reduced $\mathrm{T}_{\mathrm{H}} 2$ cytokine IL4 production under $\mathrm{T}_{\mathrm{H}} 0$ or $\mathrm{T}_{\mathrm{H}} 2$ conditions (Supplementary information, Figure S8F). Flow cytometric analysis showed that Ad-miR-151 significantly decreased $T_{H} 2$ cells under $T_{H} 0$ or $T_{H} 2$ conditions compared with Ad-GFP treatment (Supplementary information, Figure S8G). These data prompted us to examine whether osteoclast numbers rebound after MSCT as a direct result of the transplant itself (transfer of miR-151-5p). We isolated osteoclast precursors from bone marrow and induced them to differentiate into osteoclasts by treating them with M-CSF and RNAKL. The results showed that IL-4 treatment significantly inhibited osteoclastogenesis, which mimics the microenvironment in $T s k{ }^{+}$mice. Over- 

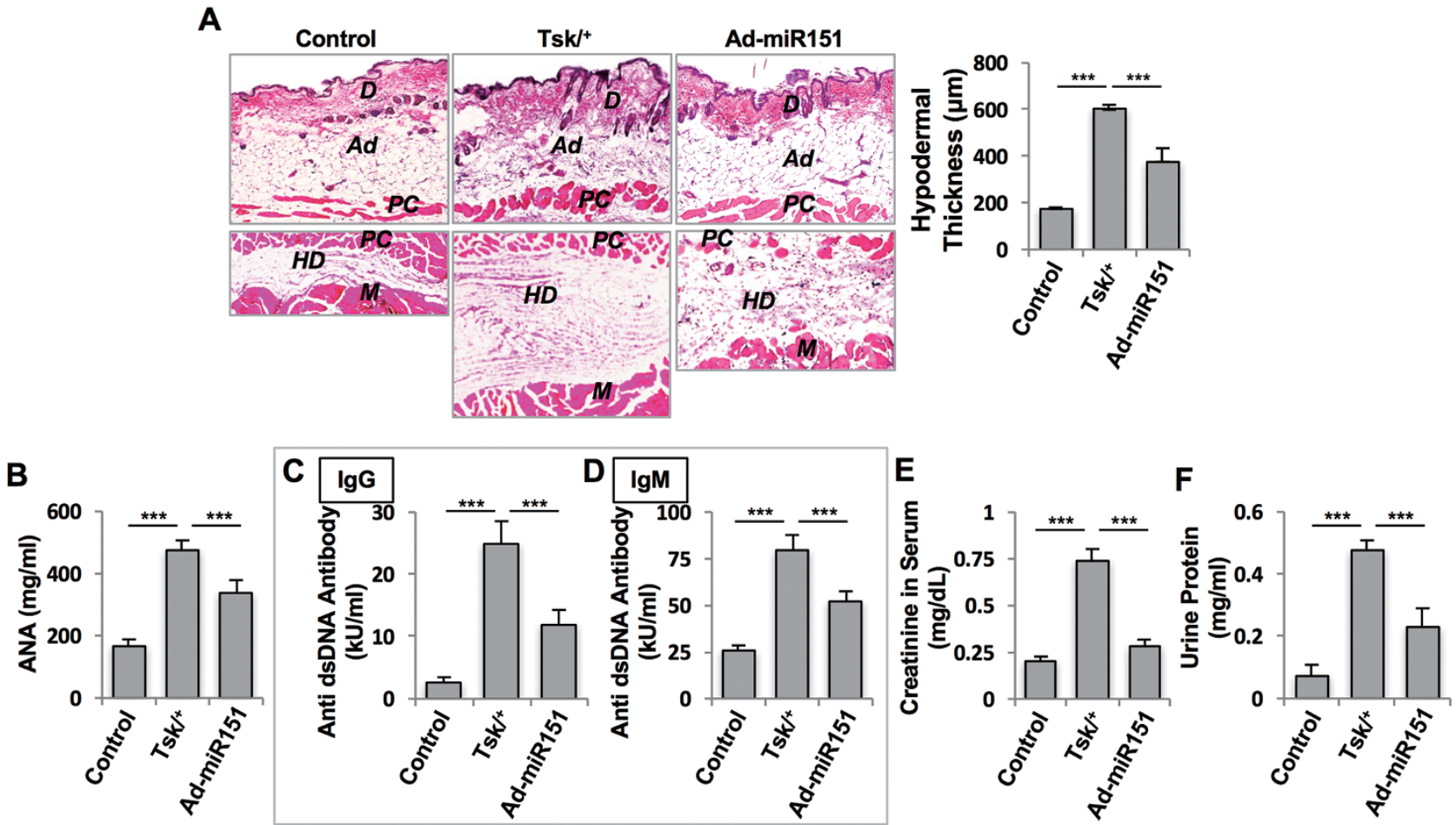

Figure 7 Ad-miR-151 treatment ameliorated skin fibrosis and autoimmune index in Tsk ${ }^{+}$mice. (A) Hypodermal thickness was significantly increased in $T s k l^{+}$mice compared to the WT group ( $n=6$ each group). Ad-miR-151 treatment significantly reduced the hypodermal thickness in Tsk $l^{+}$mice. (B-D) Tsk ${ }^{+}$mice showed elevated levels of ANA (B) and anti-double-strand DNA antibodies IgG (C) and IgM (D) when compared to control littermates. Ad-miR-151 treatment reduced the levels of ANA (B) and anti-double-strand DNA antibodies $\lg G$ (C) and IgM (D). (E) Serum creatinine levels were significantly increased in $\mathrm{Tsk}^{+}$mice. After Ad-miR-151 treatment, creatinine levels were significantly decreased to the level similar to the control group. (F) The concentration of urine protein was significantly increased in Tsk/ mice. Ad-miR-151 treatment reduced the levels of urine protein. The results were representative of three independent experiments. Ad, adipose; ANA, antinuclear antibody; D, dermis; HD, hypodermis; M, muscle; PC, panniculus carnosus. Scale bar, $50 \mu \mathrm{m} .{ }^{* * *} P<0.005$.

expression of miR151-5p with Ad-miR151 treatment inhibited IL4R $\alpha$ expression in osteoclast precursors, along with significantly increased osteoclastogenesis (Supplementary information, Figure S10). These data suggest that MSCT may directly transfer miR151-5p via EVs to increase osteoclast numbers in $T s k /^{+}$mice. Collectively, these data reveal that MSCT ameliorates disease phenotypes in $T s k /^{+}$mice through downregulating IL4R $\alpha$ signaling in recipient BMMSCs to rescue impaired osteo-/ adipogenic lineage differentiation via exosome-mediated miR-151-5p transfer (Supplementary information, Figure S11), suggesting that miR-151-5p treatment may be a target-specific therapy for SSc.

SSc patients show decreased serum levels of miR-151$5 p$ and elevated expression of IL4Ra/mTOR cascade in BMMSCS

In order to confirm that miR-151-5p-mediated downregulation of IL4R $\alpha / \mathrm{mTOR}$ signaling is relevant to SSc in human patients, we conducted a pilot clinical investigation to assess whether diminished miR-151-5p levels and activated IL4R $\alpha / \mathrm{mTOR}$ signaling can be observed in SSc patients. Twenty patients diagnosed with SSc and 20 age- and gender-matched normal controls were enrolled in this study (Supplementary information, Table S2). qPCR analysis showed that serum levels of miR-151-5p were markedly decreased in SSc patients compared to normal controls (Figure 8A). Flow cytometric analysis showed that $\mathrm{CD}^{+} \mathrm{IL}^{+} \mathrm{T}_{\mathrm{H}} 2$ cells were significantly increased in the peripheral blood of SSc patients compared to the control group (Figure 8B). ELISA further confirmed that serum IL4 levels were significantly elevated in SSc patients (Figure 8C). In addition, we found that SSc patients had significantly elevated levels of serum TGF $\beta$ and upregulation of Il4r $\alpha$ in BMMSCs by ELISA and qPCR analysis, respectively (Figure 8D and 8E). As mTOR complex 2 (TORC2) may act upstream of TORC1 in $T_{s k} /^{+}$BMMSCs [11], we showed that BMMSCs from 

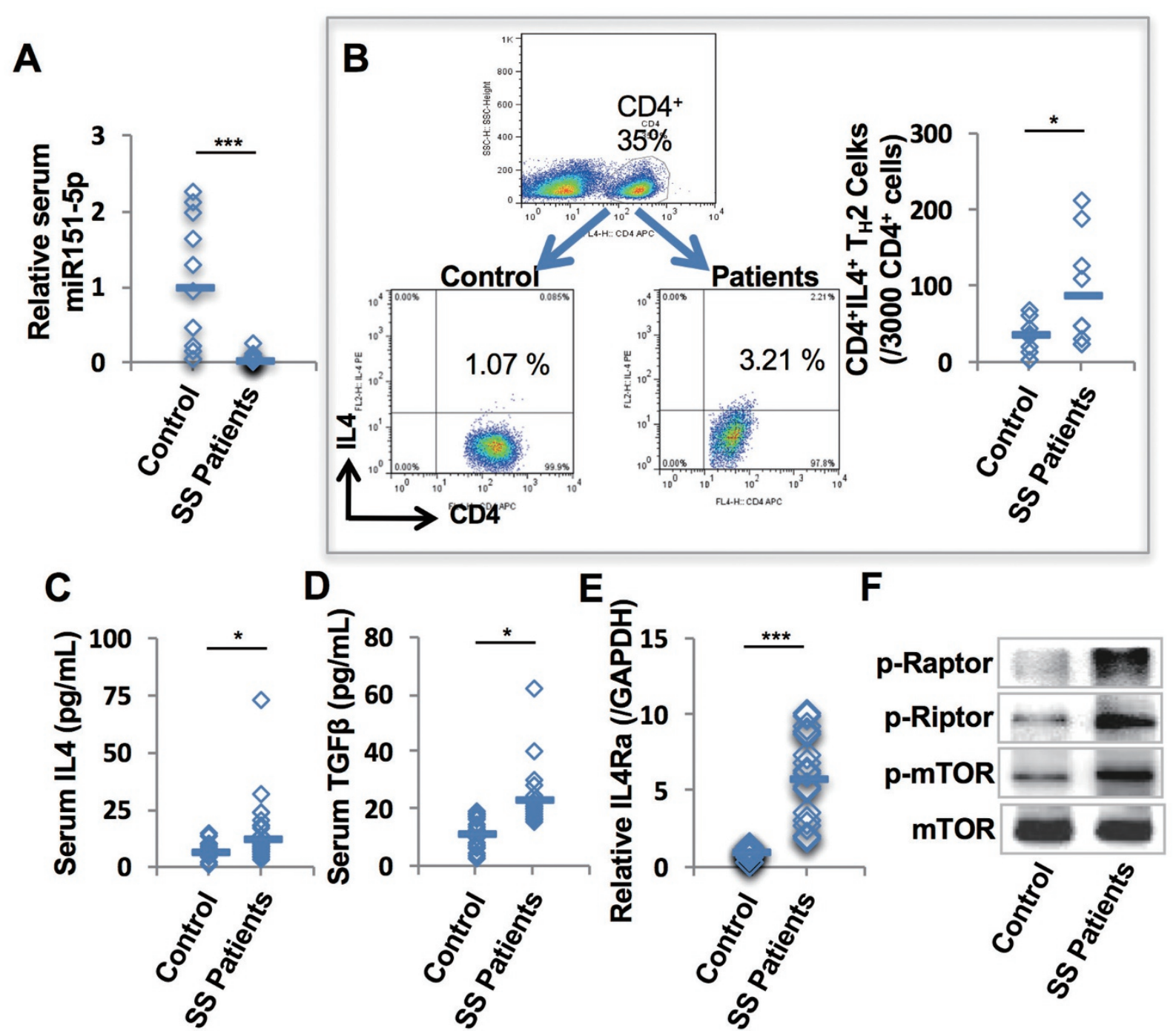

Figure 8 Systemic sclerosis patients showed decreased serum levels of miR-151-5p and elevated expression levels of IL4R $\alpha$ and mTOR in BMMSCs. (A) qPCR analysis showed the serum levels of miR-151-5p in SSc patients $(n=13)$ and normal controls $(n=11)$. (B) Flow cytometric analysis showed elevated levels of $\mathrm{T}_{\mathrm{H}} 2$ cells in peripheral blood of SSc patients compared to normal controls ( $n=9$ per group). (C) ELISA showed increased serum levels of IL4 in SSc patients compared to normal controls $(n=20)$. (D) ELISA showed that SSc patients $(n=20)$ had increased serum levels of TGF $\beta$ compared to normal controls. (E) qPCR analysis showing that BMMSCs from SSc patients $(n=20)$ had upregulated expression of $/ / 4 r \alpha$. (F) Western blot analysis showed upregulation of p-Raptor, p-Rictor, and p-mTOR in BMMSCs from SSc patients. Representative results were from three independent experiments. Error bars represent the s.d. from the mean values. ${ }^{* \star *} P<0.005 ;{ }^{*} P<0.05$.

SSc patients had upregulated expression of p-Raptor, p-Rictor, and p-mTOR (Figure 8F).

\section{Discussion}

As the first MSCT was successfully performed in severe acute GvHD patients in 2004 [12], MSCs from different sources have been widely utilized to treat various diseases [44-46]. Previous studies proposed multiple mechanisms may contribute to the success of MSCbased immune therapies such as secretion of cytokines/ chemokines, cell-cell interactions, and epigenetic regulation [24, 28, 36]. Recently, allogenic MSC-based cell therapy showed efficacy in a HOCl-induced SSc murine model, which is a closer match to the human disease [47, 48]. These studies, combined with several recent publications, suggest that the safety and efficacy of allogenic MSC-based therapy make it a promising approach in 
treating autoimmune and non-autoimmune diseases [4951]. MSCT not only rescues osteoporosis in $T_{s} \mathrm{k} /^{+}$mice, but also rescues the autoimmune phenotype and skin fibrosis in $T s k /^{+}$mice [28]. In addition, we observed that osteoclast numbers rebound after MSCT. It is possible that MSCT involves multiple mechanisms to rescue disease phenotypes in $T_{s k} /{ }^{+}$mice and other autoimmune disorders. Due to the nature of allogeneic transplant, there is little evidence to support the persistence of the graft in the recipient. Transferring miRNA and functional cellular components may be one of the therapeutic mechanisms mediated by MSCT. In this study, we showed that systemic administration of BMMSCs transfers miR-151$5 \mathrm{p}$ to $T s k /^{+}$-recipient BMMSCs to rescue impaired osteo-/ adipogenic differentiation of recipient cells. Previous studies indicated that exosomes could serve as a vehicle to transfer miRNAs between cells $[34,52]$ to regulate gene expression and have the potential to offer therapies for stroke, liver fibrosis, and cardiovascular diseases [5355]. We found that recipient $T s k /^{+}$BMMSCs are able to take miR-151-5p/exosomes from donor MSCs and reuse miR-151-5p to downregulate IL4R $\alpha$ expression, resulting in the improvement of recipient cell function. In addition, MSCs are capable of producing large amounts of exosomes [56]. Therefore, exosome/miRNA transfer may play a significant role in MSCT-mediated therapies. It appears that MSCT exerts therapeutic effects through multiple mechanisms of interaction with recipient microenvironments; however, the detailed mechanism by which the recipient microenvironment affects MSCT-mediated exosome transfer is unknown.

Differentially expressed miRNA profiles related to inflammation and fibrosis have been identified in SSc patients $[57,58]$. Previous studies suggest that miRNAs mainly modulate fibrosis-related genes such as collagens, metallopeptidases, integrins, TGF $\beta$, DNA methyltransferase 1, and toll-like receptors [59], which could partially explain why SSc phenotypes display marked heterogeneity and imply complex interplay between different pathogenic processes. Among these complex pathophysiological factors, $\mathrm{T}$ helper cell homeostasis is generally biased toward $\mathrm{T}_{\mathrm{H}} 2$ with the production of high levels of cytokines IL4 and IL13 in SSc [9]. Disruption of IL4 or IL4R $\alpha$ can rescue the fibrosis and osteopenia phenotypes, suggesting that IL4/IL4R $\alpha$ signaling may have an essential role in the disease pathogenesis [10, 11]. Accordingly, we decided to use $\mathrm{T}_{\mathrm{H}} 2$-highly activated $T s k /^{+}$mice as an SSc mouse model in this study, even though the $T s k /^{+}$model does not perfectly mimic the clinical and immunological features of human SSc. In the present study, we identified a novel target, miR-151-5p, which plays a critical role in regulating BMMSC differ- entiation via suppressing IL4R $\alpha$. Through transfection of miR151-5p mimic or adenovirus-mediated miR-151-5p overexpression, $T s k l^{+}$BMMSC function is largely improved, implying that miR-151-5p-based therapy may be a target-specific approach to treat SSc patients. Previous studies showed that multiple miRNAs are capable of regulating the osteogenic differentiation of BMMSCs and maintaining bone homeostasis $[60,61]$. Here we found that miR-151-5p mediated a lineage switch between osteogenic and adipogenic differentiation and rescued the osteopenia phenotype in $T s k /^{+}$mice. Interestingly, human miR-151-5p is able to target hIl4ra expression in human BMMSCs. Also, SSc patients showed significantly reduced serum levels of miR-151-5p, raising the possibility of translating our findings to the clinic. A major challenge in clinical practice is new biomarker discovery, which can be used for better disease diagnosis and classification. Notably, miR-151-5p may be able to serve as a biomarker for SSc diagnosis.

Gene therapy has been widely used to replace defective protein-coding genes in many genetic diseases. Given the crucial roles of miRNAs in regulating gene expression and disease development, miRNAs are considered as potential therapeutic targets for gene therapy. Recently, miRNA-based gene therapy has been established in some diseases that may be caused by the loss or reduced expression of a particular miRNA [62]. Due to the inefficient delivery or degradation of miRNAs, viral vectors encoding short hairpin RNAs, which are processed in the cell into mature miRNAs, are a promising alternative method of miRNA delivery [63]. In this study, we overexpressed miR-151 in vivo using an adenoviral vector, which produces mature miR-151-5p in recipient cells. Overexpression of miR-151-5p significantly reduced Il4r $\alpha$ expression and rescued osteopenia in $T s k l^{+}$mice, which encouraged us to examine whether adenovirus-mediated miR-151-5p overexpression could affect other organs. Indeed, SSc morbidity and mortality are strongly related to fibrosis and vasculopathy. Osteopenia may not be one of the most challenging issues in SSc. Our data indicated that Ad-miR151 treatment successfully reduced $\mathrm{T}_{\mathrm{H}} 2$ cell differentiation and decreased T-cell infiltration in the hyperdermal area, suggesting that miR-151-5p-based gene therapy can achieve therapeutic effects in multiple organs in $T s k /^{+}$mice. To carry out both effective and safe miR151-5p-based gene therapy, its potential off-target effects need to be further explored in future study.

Dysfunction of BMMSCs in $T s k{ }^{+}$mice caused by altered IL4/IL4R $\alpha$ signaling results in an osteopenia phenotype, suggesting that osteo-immunological effect on stem cell homeostasis contributes to the SSc phenotype. 
Upregulation of osteogenesis and downregulation of adipogenesis by miR-151-5p mimic treatment via inhibition of Il4ra expression may help to rebuild BMMSC function and bone marrow homeostasis. miR151-5p can serve as a promising biomarker for SSc diagnosis and a therapeutic target in SSc treatment. Given the extensive roles of MSCT in treating various autoimmune diseases, it is possible that other miRNAs can be transferred to target other specific signaling pathways.

\section{Materials and Methods}

\section{Animals}

Female C57BL/6J and B6.Cg-Fbn $1^{T_{s k}} / \mathrm{J}\left(T_{s} k /^{+}\right)$mice were purchased from Jackson Lab and maintained in a C57BL/6J background at least 10 backcrosses. Age-matched female littermates were used in all experiments. Female immunocompromised nude mice (Beige $n u / n u$ XIDIII) were purchased from Harlan. All animal experiments were performed under institutionally approved protocols for the use of animal research (University of Pennsylvania protocol number 805478 ).

\section{Isolation of mouse BMMSCs}

A single suspension of bone marrow-derived all nuclear cells (ANCs, $15 \times 10^{6}$ ) from femurs was seeded in $100 \mathrm{~mm}$ culture dishes (Corning) and incubated at $37{ }^{\circ} \mathrm{C}$ with $5 \% \mathrm{CO}_{2}$. After $48 \mathrm{~h}$, nonadherent cells were removed, and adherent cells were cultured for additional 14 days in alpha minimum essential medium ( $\alpha$-MEM, Invitrogen) supplemented with $20 \%$ fetal bovine serum (FBS), 2 $\mathrm{mM}$ L-glutamine (Invitrogen), $55 \mu \mathrm{M}$ 2-mercaptoethanol (Invitrogen), $100 \mathrm{U} / \mathrm{ml}$ penicillin, and $100 \mu \mathrm{g} / \mathrm{ml}$ streptomycin (Invitrogen). These adherent single colonies were passaged at passage one with frequent medium changes to eliminate potential hematopoietic cell contamination $[64,65]$. Since previous publications indicated that numerous passages of murine BMMSCs in atmospheric oxygen culture conditions can lead to spontaneous transformation both in vitro and in vivo [66-68], we used passage one mouse BMMSCs in this study. For CFU-F assay, $1 \times 10^{6}$ single-suspension ANCs were seeded in T25 cell culture flasks (Corning). After 16 days, the cultures were washed with PBS and stained with $1 \%$ toluidine blue solution with $2 \%$ paraformaldehyde (PFA, Sigma-Aldrich). The cell clusters were counted under microscopy and those with more than 50 cells were considered as colonies.

\section{Allogenic MSCT of SSc mice}

BMMSCs, isolated from $\mathrm{C} 57 \mathrm{BL} / 6 \mathrm{~J}$ mice at passage one, were suspended in $200 \mu \mathrm{PBS}$ and intravenously infused into $T s k /^{+} \mathrm{SSc}$ mice $\left(0.1 \times 10^{6}\right.$ cells per $10 \mathrm{~g}$ body weight $)$ at 8 weeks of age. In the control group, $T s k /^{+}$mice received PBS. Background-matched WT mice were infused with PBS intravenously as positive controls.

\section{MicroCT analysis}

After being harvested and fixed in 4\% PFA, femurs were analyzed using a high-resolution Scanco $\mu$ CT50 scanner (Scanco Medical AG, Bruttisellen, Switzerland). The specimens were scanned using a voxel size of $20 \mu \mathrm{m}$ at $70 \mathrm{kVp}$ and $200 \mu \mathrm{A}$. Datasets were analyzed using Amira 5.3.1 software (Visage Imaging, Berlin, Germany) for reconstructing images and measuring BMD.

\section{Histology}

To assess trabecular bone and bone marrow areas, femurs were fixed in 4\% PFA and then decalcified with $5 \%$ EDTA (pH 7.4), followed by paraffin embedding. Paraffin sections $6 \mathrm{~m}$ in thickness were stained with hematoxylin and eosin (H\&E) and analyzed using NIH ImageJ software. For immunohistochemistry staining, paraffin-embedded sections were incubated with the ALP or IL4 antibodies (Santa Cruz Biotechnology, Inc., 1:400) at $4{ }^{\circ} \mathrm{C}$ for overnight, and then stained with VECTASTAIN UNIVERSAL elite ABC kit and ImmPACT VIP peroxidase Substrate Kit (VECTOR) according to the manufacturer's instruction. For osteoclast activity quantification, TRAP-positive cells were identified as mature osteoclasts on the bone surface. De-paraffinized sections were re-fixed with a mixture of $50 \%$ ethanol and $50 \%$ acetone for $10 \mathrm{~min}$, and then incubated in the freshly made TRAP solution for staining with $1.6 \%$ naphthol AS-BI phosphate in $\mathrm{N}$, $\mathrm{N}$-dimethylformamide, $0.14 \%$ fast red-violet $\mathrm{LB}$ diazonium salt, $0.097 \%$ tartaric acid, and $0.04 \% \mathrm{MgCl}_{2}$ in $0.2 \mathrm{M}$ sodium acetate buffer at pH 5.0 for $10 \mathrm{~min}$ at $37{ }^{\circ} \mathrm{C}$ under a shield. Samples were counterstained with toluidine blue. All reagents for TRAP staining were purchased from Sigma-Aldrich. For double calcein labeling histomorphometric analysis, the mice were intraperitoneally injected with calcein (Sigma, $15 \mathrm{mg} / \mathrm{kg}$ body weight) prepared in $2 \%$ sodium bicarbonate solution at 10 days and 3 days before killing. Bone dynamic histomorphometric analyses for MAR and BFR/BS were performed according to the standardized nomenclature for bone histomorphometry under fluorescence microscopy (Olympus IX71, Japan).

\section{ELISA}

Peripheral blood serum was collected and analyzed using mouse ELISA Ready-SET-GO kits (eBioscience) for sRANKL, IFN $\gamma$, IL4, IL17, and IL10 protein levels, according to the manufacturer's instructions. CTX levels were analyzed with a mouse C-telopeptide of type I collagen ELISA kit (Novateinbio), according to the manufacturer's instructions. Human IL4 and TGF $\beta$ protein levels were analyzed using ELISA Ready-SET-GO kits (eBioscience), according to the manufacturer's instructions. Mouse ANA, anti-dsDNA IgG, anti-dsDNA IgM, and creatinine (Alpha Diagnosis) were measured using commercially available kits according to manufacturer's instructions.

\section{In vitro osteogenic differentiation}

BMMSCs were cultured under osteogenic inductive conditions, including $2 \mathrm{mM} \beta$-glycerophosphate (Sigma-Aldrich), 100 $\mu \mathrm{M}$ L-ascorbic acid 2-phosphate (Wako), and $10 \mathrm{nM}$ dexamethasone (Sigma-Aldrich) in the growth medium. After 3 weeks of induction, matrix mineralization was detected by $1 \%$ Alizarin Red $\mathrm{S}$ (Sigma-Aldrich) staining, and the stained positive areas were quantified using NIH ImageJ software and shown as a percentage of the total area.

\section{Co-culture of WT BMMSCs with Tsk/ $/^{+}$BMMSCs}

A transwell system for 12-well plates (Corning) was used for co-culture experiments. $T s k /^{+}$BMMSCs $\left(0.2 \times 10^{6}\right.$ cells per well $)$ were loaded in each lower chamber. WT BMMSCs $\left(0.2 \times 10^{6}\right.$ cells per well) transfected with $\mathrm{Cy} 3$ red fluorescent dye-labeled miR151-5p, Rab27a siRNA, or negative control siRNA were loaded in each upper chamber. After 3 days of co-culture, $T s k{ }^{+}$BMMSCs 
were used for microscopic imaging, total RNA isolation, protein isolation, and osteogenic differentiation analysis.

\section{Western blotting}

Cells were washed with PBS and lysed in M-PER mammalian protein extraction reagent (Thermo) with protease and phosphatase inhibitors (Roche). Protein levels were quantified using a protein concentration assay (Bio-Rad Laboratories). Twenty $\mu \mathrm{g}$ of proteins were separated by SDS-PAGE (Invitrogen) and transferred to 0.2 $\mu \mathrm{m}$ nitrocellulose membranes (Millipore). The membranes were then blocked with 5\% non-fat dry milk and $0.1 \%$ Tween-20 for $1 \mathrm{~h}$, followed by incubation overnight with primary antibodies diluted in blocking solution. Antibodies to mouse ALP (sc-28904), LPL (sc-32382), PPAR $\gamma$ (sc-7273), IL4R (sc-684), and PI3K-p110 (sc7189) were purchased from Santa Cruz Biotechnology, Inc. Antibodies to human Runx2 (sc-10758), ALP (sc-28904), and OCN (sc-18319) were purchased from Santa Cruz Biotechnology, Inc. Antibodies to phosphor-mTOR (Ser2448, 5536), mTOR (2983), phospho-Akt (Ser473, 9271), Akt (9272), phosphor-P70S6K (T389, 9243; S371, 9208), phosphor-Rictor (3806), and phosphor-Raptor (2083) were obtained from Cell Signaling Technology. Antibodies to mouse Runx2 (ab76956), OCN (Ab10911), and $\beta$-Actin (A5441) were purchased from Abcam, Millipore, and Sigma-Aldrich. The membranes were then washed and incubated for $1 \mathrm{~h}$ in HRP-conjugated secondary antibody (Santa Cruz Biotechnology, Inc.) diluted in blocking solution. Immunoreactive proteins were detected using SuperSignal West Pico Chemiluminescent Substrate (Thermo) and BioMax film (Kodak).

\section{In vivo BMMSC implantation}

$4.0 \times 10^{6} \mathrm{BMMSCs}$, isolated from C57BL/6J, Tsk/ ${ }^{+}, \mathrm{MSCT}$, exosome-treated, and Ad-miR151-treated mice, were mixed with HA/TCP ceramic powders (40 mg, Zimmer Inc.) and implanted into 8 -week-old nude mice subcutaneously. At 8 weeks post implantation, the transplants were harvested, fixed in 4\% PFA, and decalcified with $5 \%$ EDTA, followed by paraffin embedding. The $6 \mu \mathrm{m}$ paraffin sections were stained with $\mathrm{H} \& \mathrm{E}$, and bone volume was quantified by NIH ImageJ software as a percentage of the total area.

\section{Adipogenic differentiation}

For adipogenesis, BMMSCs were cultured under adipogenic inductive conditions, in growth medium containing $500 \mathrm{nM}$ isobutylmethylxanthine (Sigma-Aldrich), $60 \mu \mathrm{M}$ indomethacin (Sigma-Aldrich), $500 \mathrm{nM}$ hydrocortisone (Sigma-Aldrich), $10 \mu \mathrm{g} / \mathrm{ml}$ insulin (Sigma-Aldrich), and $100 \mathrm{nM} \mathrm{L-ascorbic} \mathrm{acid} \mathrm{phosphate.}$ At 7 days post induction, the adipocytes were stained with Oil red O (Sigma-Aldrich), and positive cells were quantified under microscopy and shown as a number out of the total cells.

\section{In vivo Oil red $O$ staining}

To quantify the adipocytes surrounding the trabecular areas, femurs were fixed in 4\% PFA and decalcified with $5 \%$ EDTA $(\mathrm{pH}$ 7.4), followed by cryosectioning and staining with Oil Red-O solution. Positive areas were quantified under microscopy and shown as a percentage of the total area.

\section{Flow cytometry}

For intercellular staining, $\mathrm{T}$ cells were stained with $1 \mu \mathrm{g}$ anti-
CD4-PerCP and CD25-APC antibodies for $30 \mathrm{~min}$ on ice. After washing and centrifuging, cells were stained with $1 \mu \mathrm{g}$ anti-IFN $\gamma$ APC, IL4-PE, IL17-PE, or Foxp3-PE antibodies using a Foxp3 staining buffer kit (eBioscience). All antibodies were purchased from BD Bioscience. After staining, all samples were analyzed using FACS ${ }^{\text {Calibur }}$ with CellQuest software (BD Bioscience).

\section{Real-time PCR}

Total RNA was isolated from the cultured cells using the miRNeasy Mini Kit (Qiagen) according to the manufacturer's instructions. Total RNA from culture medium, serum, and bone marrow supernatant was isolated using the miRNeasy Serum/Plasma Kit (Qiagen). For real-time PCR of mRNA, the cDNA was synthesized using SuperScript III (Life Technologies). The real-time PCR was performed using SYBR green supermix (Bio-Rad, Hercules, CA, USA) and gene-specific primers. For real-time PCR of mature miRNA, cDNA was synthesized using the miScript II RT Kit (Qiagen). Real-time PCR was performed using the miScript SYBR Green PCR Kit (Qiagen). RUN6 was used as an endogenous control for BMMSCs. MiR-39 was used as spike-in control for culture medium, serum, and bone marrow (Qiagen). Real-time PCR was detected on a CFX96 Real-Time PCR System (Bio-Rad).

\section{Extracellular vesicles treatment for $T_{s} k{ }^{+}$BMMSCs}

EVs were isolated from C57BL/6J murine-derived BMMSCs by ultracentrifuge. MSCs were cultured in exosome-depleted medium (complete medium depleted of FBS-derived exosomes by overnight centrifugation at $100000 \times g$ ) for $48 \mathrm{~h}$. Small EVs from culture supernatants were isolated by differential centrifugation at $300 \mathrm{~g}$ for $10 \mathrm{~min}, 2000 \times \mathrm{g}$ for $10 \mathrm{~min}, 10000 \times \mathrm{g}$ for $30 \mathrm{~min}$, and $100000 \times g$ for $70 \mathrm{~min}$ [31]. As EVs are not homogeneous [42], we used an EXOCEP exosome quantitation kit (System Biosciences Inc.) to quantitate the number of vesicles. Also, we used Exo-flow kits (System Biosciences Inc.) containing CD63 beads to show the purity of the EVs after high-speed centrifugation (Supplementary information, Figure S12). For osteogenesis assay, BMMSCs were pretreated with EVs $(20 \mu \mathrm{g} / \mathrm{ml})$ or PBS for 3 days and then cultured under osteoinductive conditions for 3 weeks, followed by Alizarin Red S staining.

\section{Extracellular vesicle treatment in $T_{s k}{ }^{+}$mice}

For in vivo EV tracking, PKH-26 (Sigma)-labeled EVs were intravenously infused into $T s k /^{+}$mice. At $24 \mathrm{~h}$ post infusion, the femurs were fixed in 4\% PFA and then decalcified with 5\% EDTA, followed by optimal cutting temperature compound (OPT, Sakura Finetek, Torrance, CA, USA) embedding. Frozen sections were prepared, and slides were mounted with Vectashield mounting medium containing DAPI (Vector Laboratories, Burlingame, CA, USA). For in vivo treatments, EVs $(100 \mu \mathrm{g})$ suspended in $200 \mu 1$ PBS or PBS (mock) were infused into $T_{s k} /^{+}$mice intravenously. Four weeks after the treatment, mice were killed for further analysis.

\section{Ad-miR-151 treatment in $T s k /^{+}$mice}

miR-151 over-expressed Adenoviral-GFP vector (Ad-miR151, Applied Biological Materials Inc.) were amplified in HEK-293 cells, followed by purification and quantification using the ViraBind Adenovirus Miniprep Kit and QuickTiter Adenovirus Quantitation kit (Cell Biolabs Inc.) according to manufacturers' instruc- 
tions. Ad-miR151 $\left(2 \times 10^{9}\right.$ p.f.u. $)$ or empty vectors were injected into the $T s k /^{+}$mice three times a week for up to 4 weeks. Samples will be harvested immediately after therapy for BMMSC isolation, histological assessments, and autoimmune index analysis. For in vivo exosome tracking, Ad-miR151 $\left(2 \times 10^{9}\right.$ p.f.u. $)$ or an empty vector was injected into the $T s k /^{+}$mice. At $24 \mathrm{~h}$ post infusion, the femurs were fixed in 4\% PFA and then decalcified with 5\% EDTA, followed by OPT embedding. Frozen sections were prepared, and slides were mounted with Vectashield mounting medium containing DAPI.

\section{T-cell purification and differentiation}

For in vitro polarization experiments, mouse $\mathrm{CD} 4{ }^{+} \mathrm{CD} 62 \mathrm{~L}^{+} \mathrm{T}$ lymphocytes were isolated from mouse total spleen cells using a magnetic sorting $\mathrm{CD}^{+} \mathrm{CD} 2 \mathrm{~L}^{+} \mathrm{T}$ cell Isolation Kit II (Miltenyi Biotec, Auburn, CA, USA), according to the manufacturer's instructions. To activate $\mathrm{T}$ cells under $\mathrm{T}_{\mathrm{H}} 0$ condition, $\mathrm{T}$ cells were activated with plate-bound anti-CD3 and anti-CD28 $(2 \mu \mathrm{g} / \mathrm{ml}$ each; BD Bioscience) in medium for 3 days. To activate $\mathrm{T}$ cells to $\mathrm{T}_{\mathrm{H}} 2$ condition, cells were activated for $72 \mathrm{~h}$ with plate-bound anti-CD3, anti-CD28, IL4 (20 ng/ml, R\&D Systems), and anti-IFN $\gamma$ neutralizing antibodies $(10 \mu \mathrm{g} / \mathrm{ml}$, Biolegend $)$.

\section{Systemic sclerosis patients}

This study was conducted in compliance with current Good Clinical Practice standards and in accordance with the principles set forth under the Declaration of Helsinki, 1989. The protocol (NCT00962923) was approved by the IRB of the Drum Tower Hospital of Nanjing University, Medical School, China. Informed consent was obtained from each patient.

\section{Statistics}

Comparisons between the two groups were analyzed using independent unpaired two-tailed Student's $t$-tests, and comparisons between more than two groups were analyzed using one-way ANOVA with the Bonferroni adjustment. $P$-values $<0.05$ were considered statistically significant.

\section{Acknowledgments}

This work was supported by grants from National Institute of Dental and Craniofacial Research, National Institutes of Health, Department of Health and Human Services (R01DE017449 and R01DE019932 to SS and K99DE025915 to CC), an NIH NIAMS fellowship (T32AR007442), and an Schoenleber Pilot Research Grant from University of Pennsylvania School of Dental Medicine. Clinical studies were supported by grants from the China Major International (Regional) Joint Project (81120108021 to LS) and Jiangsu Province Major Research and Development Program (BE 2015062 to LS). ClinicalTrials.gov Identifier: NCT00962923.

\section{Author Contributions}

SS and LS conceived the project. SS, CC, and LS designed the experiments. CC, DW, AM, DL, XK, WY, and RY performed the experiments and analyzed the data. SS and CC wrote the manuscript.

\section{Competing Financial Interests}

The authors declare that they have no competing interests.

\section{References}

1 Gabrielli A, Avvedimento EV, Krieg T. Mechanisms of disease scleroderma. N Engl J Med 2009; 360:1989-2003.

2 Omair MA, Pagnoux C, McDonald-Blumer H, Johnson SR. Low bone density in systemic sclerosis. A systematic review. $J$ Rheumatol 2013; 40:1881-1890.

3 Atteritano M, Sorbara S, Bagnato G, et al. Bone mineral density, bone turnover markers and fractures in patients with systemic sclerosis: a case control study. PLoS One 2013; 8:e66991.

4 Kilic G, Kilic E, Akgul O, Ozgocmen S. Increased risk for bone loss in women with systemic sclerosis: a comparative study with rheumatoid arthritis. Int J Rheum Dis 2016; 19:405-411.

5 Mugino H, Ikemura K. Progressive systemic sclerosis with spontaneous fracture due to resorption of the mandible: a case report. J Oral Maxillofac Surg 2006; 64:1137-1139.

6 Auluck A, Pai KM, Shetty C, Shenoi SD. Mandibular resorption in progressive systemic sclerosis: a report of three cases. Dentomaxillofac Radiol 2005; 34:384-386.

7 Souza RB, Borges CT, Takayama L, Aldrighi JM, Pereira $\mathrm{RM}$. Systemic sclerosis and bone loss: the role of the disease and body composition. Scand J Rheumatol 2006; 35:384-387.

8 Barisic-Dujmovic T, Boban I, Adams DJ, Clark SH. Marfan-like skeletal phenotype in the tight skin (Tsk) mouse. Calcif Tissue Int 2007; 81:305-315.

9 Wynn TA. Fibrotic disease and the $\mathrm{T}(\mathrm{H}) 1 / \mathrm{T}(\mathrm{H}) 2$ paradigm. Nat Rev Immunol 2004; 4:583-594.

10 Kodera T, McGaha TL, Phelps R, Paul WE, Bona CA. Disrupting the $I L-4$ gene rescues mice homozygous for the tightskin mutation from embryonic death and diminishes TGF-beta production by fibroblasts. Proc Natl Acad Sci USA 2002; 99:3800-3805.

11 Chen C, Akiyama K, Wang D, et al. IL4R $\alpha$-mediated mTOR signaling determines mesenchymal stem cell lineage differentiation in fibrillin-1-mutant-induced osteopenia. J Exp Med 2014; 212:73-91.

12 Le Blanc K, Rasmusson I, Sundberg B, et al. Treatment of severe acute graft-versus-host disease with third party haploidentical mesenchymal stem cells. Lancet 2004; 363:14391441.

13 Sun L, Akiyama K, Zhang H, et al. Mesenchymal stem cell transplantation reverses multiorgan dysfunction in systemic lupus erythematosus mice and humans. Stem Cells 2009; 27:1421-1432.

14 Liang J, Gu F, Wang H, et al. Mesenchymal stem cell transplantation for diffuse alveolar hemorrhage in SLE. Nat Rev Rheumatol 2010; 6:486-489.

15 Liu X, Zheng P, Wang X, et al. A preliminary evaluation of efficacy and safety of Wharton's jelly mesenchymal stem cell transplantation in patients with type 2 diabetes mellitus. Stem Cell Res Ther 2014; 5:57.

16 Liang J, Li X, Zhang H, et al. Allogenic mesenchymal stem cells transplantation in patients with refractory RA. Clin Rheumatol 2012; 31:157-161.

17 Hatzistergos KE, Quevedo H, Oskouei BN, et al. Bone marrow mesenchymal stem cells stimulate cardiac stem cell proliferation and differentiation. Circ Res 2010; 107:913-922. 
18 Sakaida I, Terai S, Yamamoto N, et al. Transplantation of bone marrow cells reduces CCl4-induced liver fibrosis in mice. Hepatology 2004; 40:1304-1311.

19 Yamada Y, Ueda M, Hibi H, Baba S. A novel approach to periodontal tissue regeneration with mesenchymal stem cells and platelet-rich plasma using tissue engineering technology: a clinical case report. Int $J$ Periodontics Restorative Dent 2006; 26:363-369.

20 Kishk NA, Gabr H, Hamdy S, et al. Case control series of intrathecal autologus bone marrow mesenchymal stem cell therapy for chronic spinal cord injury. Neurorehabil Neural Repair 2010; 24:702-708.

21 Liu J, Han D, Wang Z, et al. Clinical analysis of the treatment of spinal cord injury with umbilical cord mesenchymal stem cells. Cytotherapy 2013; 15:185-191.

22 Chen X, Armstrong MA, Li G. Mesenchymal stem cells in immunoregulation. Immunol Cell Biol 2006; 84:413-421.

23 Scuderi N, Ceccarelli S, Onesti MG, et al. Human adipose-derived stem cells for cell-based therapies in the treatment of systemic sclerosis. Cell Transplant 2013; 22:779-795.

24 Ren G, Zhang L, Zhao X, et al. Mesenchymal stem cell-mediated immunosuppression occurs via concerted action of chemokines and nitric oxide. Cell Stem Cell 2008; 2:141-150.

25 Choi H, Lee RH, Bazhanov N, Oh JY, Prockop DJ. Anti-inflammatory protein TSG-6 secreted by activated MSCs attenuates zymosan-induced mouse peritonitis by decreasing TLR2/NF-kappaB signaling in resident macrophages. Blood 2011; 118:330-338.

26 Meisel R, Zibert A, Laryea M, Gobel U, Daubeber W, Dilloo D. Human bone marrow stromal cells inhibit allogeneic T-cell responses by indoleamine 2,3-dioxygenase-mediated tryptophan degradation. Blood 2004; 103:4619-4621.

27 Nemeth K, Leelahavanichkul A, Yuen PS, et al. Bone marrow stromal cells attenuate sepsis via prostaglandin E(2)-dependent reprogramming of host macrophages to increase their interleukin-10 production. Nat Med 2009; 15:42-49.

28 Akiyama K, Chen C, Wang D, et al. Mesenchymal-stem -cell-induced immunoregulation involves FAS-ligand-/ FAS-mediated T cell apoptosis. Cell Stem Cell 2012; 10:544555.

29 Chen C, Akiyama K, Yamaza T, et al. Telomerase governs immunomodulatory properties of mesenchymal stem cells by regulating FAS ligand expression. EMBO Mol Med 2014; 6:322-334.

30 Hayes J, Peruzzi PP, Lawler S. MicroRNAs in cancer: biomarkers, functions and therapy. Trends Mol Med 2014; 20:460-469.

31 Thery C, Amigorena S, Raposo G, Clayton A. Isolation and characterization of exosomes from cell culture supernatants and biological fluids. Curr Protoc Cell Biol 2006; Chapter 3:Unit3.22.

32 Dreux M, Garaigorta U, Boyd B, et al. Short-range exosomal transfer of viral RNA from infected cells to plasmacytoid dendritic cells triggers innate immunity. Cell Host Microbe 2012; 12:558-570.

33 Li J, Liu K, Liu Y, et al. Exosomes mediate the cell-to cell transmission of IFN- $\alpha$-induced antiviral activity. Nat Immunol 2013; 14:793-803.

34 Luga V, Zhang L, Viloria-Petit AM, et al. Exosomes mediate stromal mobilization of autocrine Wnt-PCP signaling in breast cancer cell migration. Cell 2012; 151:1542-1556.

35 Boelens MC, Wu TJ, Nabet BY, et al. Exosome transfer from stromal to breast cancer cells regulates therapy resisitance pathways. Cell 2014; 159:499-513.

36 Liu S, Liu D, Chen C, et al. MSC transplantation improves osteopenia via epigenetic regulation of Notch signaling in lupus. Cell Metab 2015; 22:606-618.

37 Liu Y, Wang L, Liu S, et al. Transplantation of SHED prevents bone loss in the early phase of ovariectomy-induced osteoporosis. J Dent Res 2014; 93:1124-32.

38 Ono M, Kosaka N, Tominaga N, et al. Exosomes from bone marrow mesenchymal stem cells contain a microRNA that promotes dormancy in metastatic breast cancer cells. Sci Signal 2014; 7:ra63.

39 Baglio SR, Rooijers K, Koppers-Lalic D, et al. Human bone marrow- and adipose-mesenchymal stem cells secrete exosomes enriched in distinctive miRNA and tRNA species. Stem Cell Res Ther 2015; 6:127.

40 Wang B, Yao K, Huuskes BM, et al. Mesenchymal stem cells deliver exogenous microRNA-let7c via exosomes to attenuate renal fibrosis. Mol Ther 2016; 24:1290-1301.

41 Soifer HS, Rossi JJ, Saetrom P. MicroRNAs in disease and potential therapeutic applications. Mol Ther 2007; 15:20702079.

42 Witwer KW, Buzas EI, Bemis LT, et al. Standardization of sample collection, isolation and analysis methods in extracellular vesicle research. J Extracell Vesicles 2013; 2:jev. v2i0.20360.

43 Ostrowski M, Carmo NB, Krumeich S, et al. Rab27a and Rab27b control different steps of the exosome secretion pathway. Nat Cell Biol 2010; 12:19-30.

44 Nauta AJ, Fibbe WE. Immunomodulatory properties of mesenchymal stem cells. Blood 2007; 110:3499-3506.

45 Uccelli A, Moretta L, Pistoia V. Mesenchymal stem cells in health and disease. Nat Rev Immunol 2008; 8:726-736.

46 Bernardo ME, Locatelli F, Fibbe WE. Mesenchymal stromal cells. Ann NY Acad Sci 2009; 1176:101-117.

47 Maria AT, Toupet K, Bony C, et al. Antifibrotic, Antioxidant, and immunomodulatory effects of mesenchymal stem cells in HOCl-induced systemic sclerosis. Arthritis Rheumatol 2016; 68:1013-1025.

48 Maria AT, Toupet K, Maumus M, et al. Human adipose mesenchymal stem cells as potent anti-fibrosis for systemic sclerosis. J Autoimmun 2016; 70:31-39.

49 Wang D, Feng X, Lu L, et al. A CD8 T cell/indoleamine 2,3-dioxygenase axis is required for mesenchymal stem cell suppression of human systemic lupus erythematosus. Arthritis Rheumatol 2014; 66:2234-2245.

50 Golpanian S, Wolf A, Hatzistergos KE, Hare JM. Rebuilding the damaged heart: mesenchymal stem cells, cell-based therapy, and engineered heart tissue. Physiol Rev 2016; 96:11271168.

51 Mastro-Martinez I, Perez-Suarez E, Melen G, et al. Effects of local administration of allogenic adipose tissue-derived mesenchymal stem cells on functional recovery in experimental traumatic brain injury. Brain Inj 2015; 29:1497-1510.

52 Montecalvo A, Larregina AT, Shufesky WJ, et al. Mechanism of transfer of functional microRNAs between mouse dentritic 
cells via exosomes. Blood 2012; 119:756-766.

53 Xin H, Li Y, Cui Y, Yang JJ, Zhang ZG, Chopp M. Systemic administration of exosomes released from mesenchymal stromal cells promote functional recovery and neurovascular plasticity after stroke in rats. J Cereb Blood Flow Metab 2013; 33:1711-1715.

54 Li T, Yan Y, Wang B, et al. Exosomes derived from human umbilical cord mesenchymal stem cells alleviate liver fibrosis. Stem Cell Dev 2013; 15:845-854.

55 Lai RC, Chen TS, Lim SK. Mesenchymal stem cell exosome: a novel stem cell therapy for cardiovascular disease. Regen Med 2011; 6:481-492.

56 Yeo RW, Lai RC, Zhang B, et al. Mesenchymal stem cell: an efficient mass producer of exosomes for drug delivery. $A d v$ Drug Deliv Rev 2013; 65:336-341.

57 Ciechomska M, van Laar JM, O'Reilly S. Emerging role of epigenetics in systemic sclerosis pathogenesis. Genes Immun 2014; 15:433-439.

58 Jimenes SA, Piera-Velazquez S. Potential role of human-specific genes, human-specific microRNAs and human-specific non-coding regulatory RNAs in the pathogenesis of systemic sclerosis and Sjogern's syndrome. Autoimmun Rev 2013; 12:1046-1051.

59 Li Y, Huang J, Guo M, Zuo X. MicroRNAs regulating signaling pathways: potential biomarkers in systemic sclerosis. Genomics Proteomics Bioinformatics 2015; 13:234-241.

60 Clark EA, Kalomoiris S, Nolta JA, Fierro FA. Concise review: microRNA function in multipotent mesenchymal stem cells. Stem Cells 2014; 32:1074-1082.

61 Li CJ, Cheng P, Liang MK, et al. MicroRNA-188 regulates age-related switch between osteoblast and adipocyte differentiation. J Clin Invest 2015; 125:1509-1522.

62 Broderick JA, Zamore PD. MicroRNA therapeutics. Gene Therapy 2011; 18:1104-1110.

63 Bonci D, Coppola V, Musumeci M, et al. The miR15a-miR16-1 cluster controls prostate cancer by targeting multiple oncogenic activities. Nat Med 2008; 14:1271-7.

$64 \mathrm{Zhu} \mathrm{H}$, Guo ZK, Jiang XX, et al. A protocol for isolation and culture of mesenchymal stem cells from mouse compact bone. Nat Protoc 2010; 5:550-560.

65 Soleimani M, Nadri S. A protocol for isolation and culture of mesenchymal stem cells from mouse bone marrow. Nat Protoc 2009; 4:102-106.

66 Miura M, Miura Y, Padilla-Nash HM, et al. Accumulated chromosomal instability in murine bone marrow mesenchymal stem cells leads to malignant transformation. Stem Cells 2006; 24:1095-1103.

67 Boregowda SV, Krishnappa V, Chambers JW, et al. Atmospheric oxygen inhibits growth and differentiation of marrow-derived mouse mesenchymal stem cells via a p53-dependent mechanism: implications for long-term culture expansion. Stem Cells 2012; 30:975-987.

68 Phinney DG. Functional heterogeneity of mesenchymal stem cells: implications for cell therapy. J Cell Biochem 2012; 113:2806-2812.

(Supplementary information is linked to the online version of the paper on the Cell Research website.) 